\title{
Regular 1-harmonic flow
}

\author{
Lorenzo Giacomelli $^{1} \cdot$ Michał Łasica ${ }^{1,2} \cdot$ Salvador Moll ${ }^{3}$
}

Received: 15 January 2018 / Accepted: 25 February 2019 / Published online: 20 April 2019

(c) The Author(s) 2019

\begin{abstract}
We consider the 1-harmonic flow of maps from a bounded domain into a submanifold of a Euclidean space, i.e., the gradient flow of the total variation functional restricted to maps taking values in the manifold. We restrict ourselves to Lipschitz initial data. We prove uniqueness and, in the case of a convex domain, local existence of solutions to the flow equations. If the target manifold has non-positive sectional curvature or in the case that the datum is small, solutions are shown to exist globally and to become constant in finite time. We also consider the case where the domain is a compact Riemannian manifold without boundary, thus solving the homotopy problem for 1-harmonic maps under some assumptions on both manifolds.
\end{abstract}

Mathematics Subject Classification 35K51 - 35A01 - 35A02 - 35B40 - 35D35 - 35K92 . $35 \mathrm{R} 01 \cdot 53 \mathrm{C} 21 \cdot 68 \mathrm{U} 10$

\section{Contents}

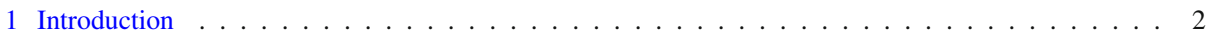

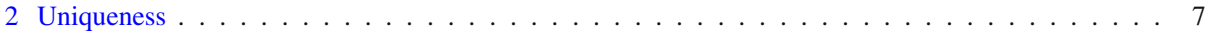

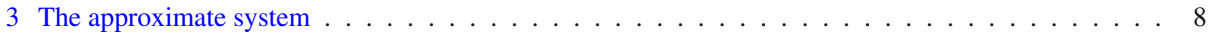

3.1 Uniform bounds . . . . . . . . . . . . . . . . . . . . . 8

3.2 Existence for the approximate system . . . . . . . . . . . . . . . . . . 11

4 Local existence . . . . . . . . . . . . . . . . . . . . . . . . . . 13

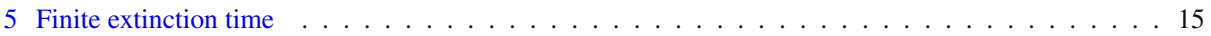

Communicated by Y. Giga.

$\triangle$ Michał Łasica

lasica@mimuw.edu.pl

Lorenzo Giacomelli

lorenzo.giacomelli@sbai.uniroma1.it

Salvador Moll

j.salvador.moll@uv.es

1 SBAI Department, Sapienza University of Rome, Via Antonio Scarpa, 16, 00161 Rome, Italy

2 Institute of Applied Mathematics and Mechanics, University of Warsaw, Banacha 2, 02-097 Warsaw, Poland

3 Department of Mathematical Analysis, University of Valencia, C/Dr. Moliner, 50, 46100 Burjassot, Spain 
6 Non-positive sectional curvature of the target . . . . . . . . . . . . . . . . . . . 17

7 The case where the domain is a Riemannian manifold . . . . . . . . . . . . . . . . . 18

Appendix: Technical lemmata . . . . . . . . . . . . . . . . . . . . 21

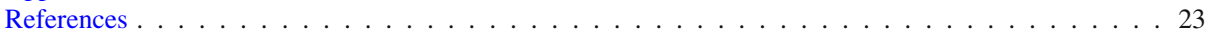

\section{Introduction}

Let $(\mathscr{N}, g)$ be a complete, connected smooth $n$-dimensional Riemannian manifold (without boundary). Throughout the paper, without loss of generality [21,34], we will treat it as an isometrically embedded submanifold in the Euclidean space $\mathbb{R}^{N}$. Given an open, bounded Lipschitz domain $\Omega \subset \mathbb{R}^{m}$ we consider the formal steepest descent flow with respect to the $L^{2}$ distance of the functional $\operatorname{TV}_{\Omega}^{\mathscr{N}}$ : the total variation functional constrained to functions taking values in $\mathscr{N}$, given for smooth $\mathbf{u}$ by

$$
\mathrm{TV}_{\Omega}^{\mathscr{N}}[\mathbf{u}]=\int_{\Omega}|\nabla \mathbf{u}|
$$

Following the $L^{2}$-steepest descent flow is one way of controlled decreasing $\mathrm{TV}_{\Omega}^{\mathcal{N}}$, which is a problem appearing in image processing. Besides the case $\mathscr{N} \subseteq \mathbb{S}^{N-1}$, which appears in denoising of optical flows [40] or color images [41], other examples of targets appearing in applications include the space of isometries $S O(3) \times \mathbb{R}^{3}[28]$, the cylinder $\mathbb{R}^{2} \times \mathbb{S}^{1}$ (LCh color space) [45] and the space of positive definite symmetric matrices (diffusion tensors) Sym $_{+}$(3) [45]. All of these examples are homogeneous spaces, and therefore have natural invariant metrics. Our main goal in this paper is to develop a well-posedness theory for the flow in a generality encompassing these cases. As some of these manifolds are non-compact, we refrain from the unnecessary (although convenient) assumption of compactness of $\mathscr{N}$.

Given a point $\mathbf{p} \in \mathscr{N}$, we denote by

$$
\pi_{\mathbf{p}}: T_{\mathbf{p}} \mathbb{R}^{N} \equiv \mathbb{R}^{N} \rightarrow T_{\mathbf{p}} \mathscr{N}
$$

the orthogonal projection onto the tangent space of $\mathscr{N}$ at $\mathbf{p}, T_{\mathbf{p}} \mathscr{N}$. Similarly, $\pi_{\mathbf{p}}^{\perp}$ will denote the orthogonal projection of $\mathbb{R}^{N}$ onto the normal space $T_{\mathbf{p}} \mathscr{N}^{\perp}$. The centered dot will denote the Euclidean scalar product on $\mathbb{R}^{m}$ or $\mathbb{R}^{N}$, while $k$ stacked dots will denote the induced scalar product on a Cartesian product of any $k$-tuple of these spaces. Calculating the first variation of (1) at $\mathbf{u}$, one obtains that the flow in a time interval [0, $T$ [ starting with initial datum $\mathbf{u}_{0}$ is formally given by the system

$$
\begin{aligned}
& \left.\mathbf{u}_{t}=\pi_{\mathbf{u}}\left(\operatorname{div} \frac{\nabla \mathbf{u}}{|\nabla \mathbf{u}|}\right) \quad \text { in }\right] 0, T[\times \Omega, \\
& \left.\boldsymbol{v}^{\Omega} \cdot \frac{\nabla \mathbf{u}}{|\nabla \mathbf{u}|}=\mathbf{0} \text { in }\right] 0, T[\times \partial \Omega, \\
& \mathbf{u}(0, \cdot) \equiv \mathbf{u}_{0} .
\end{aligned}
$$

The symbol $\boldsymbol{v}^{\Omega}$ denotes the external unit normal of $\Omega$, which is defined $\mathscr{H}^{m-1}$-a.e., on $\partial \Omega$. The meaning of the expression $\frac{\nabla \mathbf{u}}{|\nabla \mathbf{u}|}$ in (2), (3) deserves a clarification even for smooth $\mathbf{u}$ : we understand $\frac{\nabla \mathbf{u}}{|\nabla \mathbf{u}|}$ as a multifunction

$$
\frac{\nabla \mathbf{u}}{|\nabla \mathbf{u}|}:(t, \mathbf{x}) \mapsto \begin{cases}\frac{\nabla \mathbf{u}(t, \mathbf{x})}{|\nabla \mathbf{u}(t, \mathbf{x})|} & \text { if } \nabla \mathbf{u}(t, \mathbf{x}) \neq 0 \\ B(0,1) \subset \mathbb{R}^{m} \times T_{\mathbf{u}(t, \mathbf{x})} \mathscr{N} & \text { if } \quad \nabla \mathbf{u}(t, \mathbf{x})=0\end{cases}
$$


and require that (2), (3) are satisfied for an appropriate selection. This is formalized in the following definition, which is an adapted version of [3, Definition 2.5]. Here and in the following we will use the notation

$$
X(U, \mathscr{N})=\left\{\mathbf{w} \in X\left(U, \mathbb{R}^{N}\right): \mathbf{w}(\mathbf{y}) \in \mathscr{N} \text { for a. e. } \mathbf{y} \in U\right\},
$$

where $U$ is any domain in $\mathbb{R}^{l}$ (or a compact $l$-dimensional Riemannian manifold), $l=1,2, \ldots$ and $X\left(U, \mathbb{R}^{N}\right)$ is a subspace of $L_{l o c}^{1}\left(U, \mathbb{R}^{N}\right)$.

Definition 1 Let $T \in] 0, \infty]$. We say that

$$
\mathbf{u} \in W_{l o c}^{1,2}\left(\left[0 , T [ \times \overline { \Omega } , \mathscr { N } ) \text { with } \nabla \mathbf { u } \in L _ { l o c } ^ { \infty } \left(\left[0, T\left[\times \bar{\Omega}, \mathbb{R}^{m \cdot N}\right)\right.\right.\right.\right.
$$

is a (regular) solution to (2) (in $\left[0, T\left[\right.\right.$ ) if there exists $Z \in L^{\infty}(] 0, T\left[\times \Omega, \mathbb{R}^{m \cdot N}\right.$ ) with $\operatorname{div} \mathrm{Z} \in L_{\text {loc }}^{2}\left(\left[0, T\left[\times \bar{\Omega}, \mathbb{R}^{N}\right)\right.\right.$ satisfying

$$
\begin{aligned}
\mathbf{Z} & \in \frac{\nabla \mathbf{u}}{|\nabla \mathbf{u}|}, \\
\mathbf{u}_{t} & =\pi_{\mathbf{u}}(\operatorname{div} \mathbf{Z})
\end{aligned}
$$

$\mathscr{L}^{1+m}-$ a. e. in $] 0, T[\times \Omega$. We say that a regular solution $\mathbf{u}$ to (2) satisfies (homogeneous) Neumann boundary condition (3) if

$$
v^{\Omega} \cdot \mathrm{Z}=\mathbf{0}
$$

$\mathscr{L}^{1} \otimes \mathscr{H}^{m-1}-$ a. e. in $] 0, T[\times \partial \Omega$.

Remark 1 Due to Morrey embedding theorem, any regular solution to (2) has a representative that is locally Hölder continuous on $[0, T[\times \bar{\Omega}$ [22, Theorem 5]. We will identify it with this representative. In particular, the initial condition (4) can be understood pointwise. On the other hand, $\boldsymbol{v}^{\Omega} \cdot \mathrm{Z}$ in (7) has to be understood as the normal trace of an $L^{\infty}$ vector field with integrable divergence, as defined in $[4,42]$.

If conditions in Definition 1 are satisfied, we will often say that the pair (u, Z) is a (regular) solution to (2) and/or (3). We will often use equivalent (see e.g., the proof of Lemma 2) form of (6):

$$
\mathbf{u}_{t}=\operatorname{div} \mathbf{Z}+\mathscr{A}_{\mathbf{u}}\left(\mathbf{u}_{x^{i}}, \mathbf{Z}_{i}\right),
$$

where $\mathscr{A}_{\mathbf{p}}$ denotes the second fundamental form of $\mathscr{N}$ at $\mathbf{p} \in \mathscr{N}$ and $\mathbf{Z}=\left(\mathbf{Z}_{1}, \ldots, \mathbf{Z}_{m}\right)$. Here and throughout the paper, we use Einstein's summation convention.

The adjective regular in Definition 1 is justified by the following considerations. Firstly, $W^{1, \infty}(\Omega)$ is the highest Sobolev regularity that is preserved by the scalar total variation flow $[5,25]$. Secondly, such attribute distinguishes the class of solutions in Definition 1 from weak (energy) solutions, whose natural spatial regularity is $B V(\Omega)$. However, we note that in the constrained case, even defining a proper concept of solution is non-trivial in the $B V$ setting, the crucial issue being an appropriate identification of the right-hand side of (6) or of (8). In this regard, the only case considered so far is $\mathscr{N} \subseteq \mathbb{S}^{n}$, in which (8) drastically simplifies due to the isotropy of the sphere:

$$
\mathbf{u}_{t}=\operatorname{div} \mathbf{Z}+\mathbf{u}|\nabla \mathbf{u}| .
$$

Suitably defined solutions to (2), (3) have been obtained in [15] when the initial datum is contained in an hyper-octant of $\mathbb{S}^{n}[15]$. When $n=1$, the assumption on $u_{0}$ may be relaxed and uniqueness results are available too [14]. A notion of solution extending the one in $[14,15]$ 
to $(N-1)$-dimensional manifolds with unique geodesics has been proposed in [9]. Existence of solutions for a discretized Dirichlet problem for (2) in the case $\mathscr{N}=\mathbb{S}^{n}, m=2$ has been obtained in [20]. The validity of Definition 1 is supported by the well-posedness results that we obtain. First of all, regular solutions are unique.

Theorem 1 Suppose that $\mathbf{u}^{1}, \mathbf{u}^{2}$ are two regular solutions to (2), (3) in $[0, T[, T \in] 0, \infty[$ such that $\mathbf{u}^{1}(0, \cdot)=\mathbf{u}^{2}(0, \cdot)=\mathbf{u}_{0}$. Then $\mathbf{u}^{1} \equiv \mathbf{u}^{2}$.

The proof Theorem 1 is different from the proofs of analogous results for $p$-harmonic flow in $[12,23]$ in that we do not use strict monotonicity of the $p$-Laplace operator (since it does not hold for $p=1$ ).

Provided that $\Omega$ is convex, we are able to construct local-in-time Lipschitz solutions to (2), (3). We need the assumption of convexity, as we are forced to use global $L^{p}$ estimates for $\nabla \mathbf{u}$. Localization of these estimates is not available due to the strong degeneracy of the 1-Laplace operator div $\frac{\nabla \mathbf{u}}{|\nabla \mathbf{u}|}$. In fact, at least in the case of anisotropic total variation flow, there are examples of non-convex Lipschitz $\Omega$, where $W^{1, p}(\Omega)$ regularity classes are not preserved by the flow [27]. The assumption of convexity is not very restrictive from the point of view of image processing, as typical domains in applications are rectangles (or boxes of different dimensions).

The existential theory depends on the sectional curvature $\mathscr{K}_{\mathscr{N}}$ of $\mathscr{N}$ or, equivalently, on the Riemannian curvature tensor $\mathscr{R}^{\mathscr{N}}$ of $\mathscr{N}$. We denote by $K_{\mathscr{N}}$ the supremum of sectional curvature over $\mathscr{N}$, i.e.,

$$
K_{\mathscr{N}}=\sup \left\{\frac{\mathbf{v} \cdot \mathscr{R}_{\mathbf{p}}^{\mathscr{N}}(\mathbf{v}, \mathbf{w}) \mathbf{w}}{|\mathbf{v}|^{2}|\mathbf{w}|^{2}-(\mathbf{v} \cdot \mathbf{w})^{2}} \mid \mathbf{p} \in \mathscr{N}, \mathbf{v}, \mathbf{w} \in T_{\mathbf{p}} \mathscr{N} \text { linearly independent }\right\} .
$$

Recall that $K_{S O(n) \times \mathbb{R}^{n}}$ is positive (and finite) and $K_{\mathbb{S}^{1} \times \mathbb{R}^{n}}, K_{S y m_{+}(n)}$ are non-positive.

Theorem 2 Suppose that $\Omega$ is convex, the embedding of $\mathscr{N}$ in $\mathbb{R}^{N}$ is closed and $K_{\mathscr{N}}<\infty$. Given $\mathbf{u}_{0} \in W^{1, \infty}(\Omega, \mathscr{N})$, we denote $T_{\dagger}=\left(K_{\mathscr{N}}\left\|\nabla \mathbf{u}_{0}\right\|_{L^{\infty}}\right)^{-1}$ if $K_{\mathscr{N}}>0$ and $T_{\dagger}=$ $+\infty$ otherwise. There exists a regular solution $\mathbf{u}$ to (2)-(4) in $\left[0, T_{\dagger}[\right.$ satisfying the energy inequality

$$
\operatorname{ess} \sup _{t \in\left[0, T_{\dagger}[\right.} \int_{\Omega}|\nabla \mathbf{u}(t, \cdot)|+\int_{0}^{T_{\dagger}} \int_{\Omega} \mathbf{u}_{t}^{2} \leq \int_{\Omega}\left|\nabla \mathbf{u}_{0}\right| .
$$

This theorem bears a similarity to [17, Theorem 3.4], where Lipschitz local-in-time solutions to (2) are constructed in the case where $\Omega$ is a flat torus, i.e., a box with periodic boundary conditions. However, aside from the choice of boundary condition, there are differences between these results - most importantly, in [17], smallness of $\nabla \mathbf{u}_{0}$ in $L^{1+\varepsilon}(\Omega)$ is assumed. This is because in [17], global solutions to $p$-harmonic flows constructed in [13] for small initial data are used as an approximation. In our case a different approximation scheme is proposed. In fact we cannot use the results in [13] as non-trivial boundary conditions are not handled there.

At least in the case of Dirichlet boundary data, regular solutions to (2) can blow up in finite time, as shown by explicit examples in $[8,16]$. In our case, we prove that solutions exist globally in time, provided that the range of the initial datum is contained in a small enough ball in $\mathscr{N}$. In fact, in this case they become constant in finite time, similarly as for the scalar total variation flow [18]. Note that in the case of inhomogeneous Dirichlet boundary conditions, the evolution of generic initial data under 1-harmonic flow does not stop in finite time [19], 
in contrast to what is observed in the scalar total variation flow, at least in 1-dimensional domains [25]. Let us denote by $B_{g}(\mathbf{p}, R)$ the ball centered at $\mathbf{p} \in \mathscr{N}$ of radius $R>0$ with respect to the metric induced by $g$ on $\mathscr{N}$.

Theorem 3 Let $\mathbf{p}_{0} \in \mathscr{N}, \mathbf{u}_{0} \in W^{1, \infty}(\Omega, \mathscr{N})$ and $\mathbf{u}$ be a regular solution to (2)-(4) in $[0, T[$. Suppose that $\mathbf{u}_{0}(\Omega) \in \overline{B_{g}\left(\mathbf{p}_{0}, R\right)}, R>0$. There exist

- a constant $R_{*}=R_{*}\left(\mathscr{N}, \mathbf{p}_{0}\right)>0$ such that if $R<R_{*}$, then $\mathbf{u}(t, \Omega) \in \overline{B_{g}\left(\mathbf{p}_{0}, R\right)}$ for $t \in] 0, T[$,

- constants $\left.\widetilde{R}_{*}=\widetilde{R}_{*}\left(\mathscr{N}, \mathbf{p}_{0}\right) \in\right] 0, R_{*}\left[, C=C\left(\Omega, \mathscr{N}, \mathbf{p}_{0}\right)>0\right.$ and $\mathbf{u}_{*} \in \mathscr{N}$ such that if $R<\min \left(\widetilde{R}_{*}, \frac{T}{C}\right)$, then $\mathbf{u}(t, \cdot) \equiv \mathbf{u}_{*}$ for $\left.t \in\right] C R, T[$.

In the particular case $K_{\mathscr{N}} \leq 0$ no blow-up occurs for any Lipschitz datum, and we can obtain a stronger result of global existence. Owing to particularly simple topology of Riemannian manifolds with $K_{\mathscr{N}} \leq 0$, we need not assume the existence of a closed embedding of $\mathscr{N}$ into $\mathbb{R}^{N}$ in this case.

Theorem 4 Suppose that $\Omega$ is convex and $K_{\mathscr{N}} \leq 0$. Let $\mathbf{u}_{0} \in W^{1, \infty}(\Omega, \mathscr{N})$. There exists a regular solution $\mathbf{u}$ to (2)-(4) in [0, $\infty$ [ satisfying the energy inequality (10). There exists $T_{*}=$ $T_{*}\left(\mathbf{u}_{0}\right) \in\left[0, \infty\left[\right.\right.$ and $\mathbf{u}_{*}=\mathbf{u}_{*}\left(\mathbf{u}_{0}\right) \in \mathscr{N}$ such that $\mathbf{u}(t, \cdot) \equiv \mathbf{u}_{*}$ for $t \geq T_{*}$. Furthermore,

$$
\text { ess } \sup _{t>0}\|\nabla \mathbf{u}(t, \cdot)\|_{L^{\infty}(\Omega)} \leq\left\|\nabla \mathbf{u}_{0}\right\|_{L^{\infty}(\Omega)} .
$$

We remark that in the scalar case the preservation of the $W^{1, \infty}$ bound follows from [7, Corollary 5.6]. However, the methods there are not readily adaptable to vectorial problems.

From the point of view of imaging science, the rigorously defined notion of regular solution to (2)-(4) provides a theoretical basis for computing a total variation diminishing flow via a finite difference scheme. Our well-posedness results should then be expected to translate to stability results for such a scheme. In these terms, the requirement of Lipschitz regularity of data is not a significant restriction, as it corresponds to the boundedness of difference quotients on the level of discretization. In the case where $\mathscr{N}$ has non-positive sectional curvature (Theorem 4), stability propagates indefinitely, even if initial image exhibits prominent contours. However, this is not necessarily the case anymore for general $\mathscr{N}$, as the bound on existence time $T_{\dagger}$ of the solution constructed in Theorem 2 deteriorates with increasing Lipschitz constant of the datum. For this reason, well-posedness for a notion of solution defined on the energy space $B V(\Omega, \mathscr{N})$ would be more desirable. In fact, we plan to use the present paper as a basis for treating this problem.

The regular 1-harmonic flow that we consider here is continuous over the spacetime, and hence capable of generating homotopy. For this reason we find it appropriate to discuss in detail the case where the domain is a compact Riemannian manifold $(\mathscr{M}, \gamma)$. In this setting, the total variation functional takes form

$$
\mathrm{TV}_{\mathscr{M}}^{\mathscr{N}}[\mathbf{u}]=\int_{\mathscr{M}}|\mathrm{d} \mathbf{u}|_{\gamma} \mathrm{d} \mu_{\gamma} .
$$

To explain the notation in (11), we introduce local coordinates $\mathbf{x} \mapsto\left(x^{1}, \ldots, x^{m}\right)$ on $\mathscr{M}$ and denote $\gamma(\mathbf{v}, \mathbf{w})=\gamma_{a b} v^{a} w^{b}$ for any vector fields $\mathbf{v}, \mathbf{w}$ on $\mathscr{M},\left(\gamma^{a b}\right)_{1 \leq a, b \leq m}=\left(\gamma_{a b}\right)_{1 \leq a, b \leq m}^{-1}$. We have $|\mathrm{d} \mathbf{u}|_{\gamma}=\left(\gamma^{a b} u_{x^{a}}^{i} u_{x^{b}}^{i}\right)^{\frac{1}{2}}$ and $\mathrm{d} \mu_{\gamma}=\left|\operatorname{det}\left(\gamma_{a b}\right)\right|^{\frac{1}{2}} \mathrm{~d} \mathscr{L}^{m}$. In this setting, the system of Eq. (2) representing the flow becomes 


$$
\left.\mathbf{u}_{t}=\pi_{\mathbf{u}}\left(\operatorname{div}_{\gamma} \frac{\mathrm{du}}{|\mathrm{du}|}\right) \quad \text { in }\right] 0, T[\times \mathscr{M} .
$$

The expression for $\operatorname{div}_{\gamma}$ acting on a 1 -form $\vartheta$ on $\mathscr{M}$ in coordinates is

$$
\operatorname{div}_{\gamma} \vartheta=\left|\operatorname{det}\left(\gamma_{a b}\right)\right|^{-\frac{1}{2}}\left(\left|\operatorname{det}\left(\gamma_{a b}\right)\right|^{\frac{1}{2}} \gamma^{a b} \vartheta_{b}\right)_{x^{a}} .
$$

Observe that (12) is a formal limit as $p \rightarrow 1^{+}$of systems

$$
\left.\mathbf{u}_{t}=\pi_{\mathbf{u}}\left(\operatorname{div}_{\gamma}\left(|\mathrm{d} \mathbf{u}|^{p-2} \mathrm{du}\right)\right) \quad \text { in }\right] 0, T[\times \mathscr{M}
$$

corresponding to $p$-harmonic map flows between Riemannian manifolds. These were first considered in the case $p=2$ in connection with the homotopy problem for harmonic maps, i.e., the problem of finding a harmonic map homotopic to a given one. The problem was solved in [11] under the condition that $K_{\mathscr{N}} \leq 0$ by constructing the harmonic map flow. An analogous result was later obtained in [12] for any $p>1$. We note that there are several non-equivalent notions of $p$-harmonic maps, among them weakly p-harmonic maps, i.e., stationary weak solutions to (13).

We introduce the notation

$$
\frac{\mathrm{du}}{|\mathrm{d} \mathbf{u}|_{\gamma}}:(t, \mathbf{x}) \mapsto\left\{\begin{array}{lll}
\frac{\mathrm{d} \mathbf{u}(t, \mathbf{x})}{|\mathrm{du}(t, \mathbf{x})|_{\gamma}} & \text { if } & \mathrm{d} \mathbf{u}(t, \mathbf{x}) \neq 0 \\
B_{\gamma}(0,1) \subset T_{\mathbf{x}}^{*} \mathscr{M} \times T_{\mathbf{u}(t, \mathbf{x})} \mathscr{N} & \text { if } & \mathrm{d} \mathbf{u}(t, \mathbf{x})=0 .
\end{array}\right.
$$

Measurable selections of $\frac{\mathrm{d} \mathbf{u}}{|\mathrm{d} \mathbf{u}|_{\gamma}}(t, \cdot)$ can be seen as $L^{\infty}$ sections of the bundle $T^{*} \mathscr{M} \times \mathbb{R}^{N}$ over $\mathscr{M}$ for a.e., $t \in] 0, T$ [, see [35] for reference. As in [35], we let $L^{p}\left(T^{*} \mathscr{M} \times \mathbb{R}^{N}\right)$ denote $L^{p}$ sections of this bundle, $p \in[1, \infty]$. Similarly, we denote by $L^{p}(] 0, T\left[\times T^{*} \mathscr{M} \times\right.$ $\mathbb{R}^{N}$ ) the space of $L^{p}$ sections of the bundle $] 0, T\left[\times T^{*} \mathscr{M} \times \mathbb{R}^{N}\right.$ over $] 0, T[\times \mathscr{M}$, and by $L_{\text {loc }}^{p}\left(\left[0, T\left[\times T^{*} \mathscr{M} \times \mathbb{R}^{N}\right)\right.\right.$ the space of measurable sections of this bundle which are $p$ integrable locally on $[0, T[\times \mathscr{M}$. We are ready to introduce a concept of solution to (12).

Definition 2 Let $T \in] 0, \infty]$. We say that

$$
\mathbf{u} \in W_{l o c}^{1,2}\left(\left[0 , T [ \times \mathscr { M } , \mathscr { N } ) \quad \text { with } \quad \mathrm { d } \mathbf { u } \in L _ { \text { loc } } ^ { \infty } \left(\left[0, T\left[\times T^{*} \mathscr{M} \times \mathbb{R}^{N}\right)\right.\right.\right.\right.
$$

is a (regular) solution to (12) (in [0,T [) if there exists $Z \in L^{\infty}(] 0, T\left[\times T^{*} \mathscr{M} \times \mathbb{R}^{N}\right)$ with $\operatorname{div}_{\gamma} Z \in L_{l o c}^{2}\left(\left[0, T\left[\times \mathscr{M}, \mathbb{R}^{N}\right)\right.\right.$ satisfying

$$
\begin{aligned}
\mathbf{Z} & \in \frac{\mathrm{d} \mathbf{u}}{|\mathrm{d} \mathbf{u}|_{\gamma}}, \\
\mathbf{u}_{t} & =\pi_{\mathbf{u}}\left(\operatorname{div}_{\gamma} \mathbf{Z}\right)
\end{aligned}
$$

$\mathscr{L}^{1+m}-$ a. e. in $] 0, T[\times \mathscr{M}$.

The strength of our result in this case depends on the sign of the Ricci curvature $\mathscr{R} i c^{\mathscr{M}}$ of $\mathscr{M}$. Opposite to the usual convention, we define it as a $(2,0)$ tensor, i.e.,

$$
\left(\mathscr{R i} c^{\mathscr{M}}\right)^{a b}=\gamma^{a c} \gamma^{b d}\left(\mathscr{R}^{\mathscr{M}}\right)_{c e d}^{e}
$$

in coordinates. We denote

$$
\operatorname{Ric}_{\mathscr{M}}=\min \left\{\frac{\mathscr{R} i c_{\mathbf{p}}^{\mathscr{M}}(\boldsymbol{\vartheta}, \boldsymbol{\eta})}{|\boldsymbol{\vartheta}|_{\gamma}|\boldsymbol{\eta}|_{\gamma}} \mid \mathbf{p} \in \mathscr{M}, \boldsymbol{\vartheta}, \boldsymbol{\eta} \in T_{p}^{*} \mathscr{M} \backslash\{\boldsymbol{0}\}\right\} .
$$


Theorem 5 Let $(\mathscr{M}, \gamma)$ be a compact, orientable Riemannian manifold and let $(\mathscr{N}, g)$ be a compact submanifold in the Euclidean space $\mathbb{R}^{N}$. Given $\mathbf{u}_{0} \in W^{1, \infty}(\mathscr{M}, \mathscr{N})$, there exists $T \in] 0, \infty]$ and a unique regular solution to (12), (4) in $[0, T[$.

If $K_{\mathscr{N}} \leq 0$, the solution exists in $[0, \infty[$. If in addition Ric $\mathscr{M} \geq 0$, there exists a sequence $\left.\left(t_{k}\right) \subset\right] 0, \infty\left[, t_{k} \rightarrow \infty, \mathbf{u}_{*} \in W^{1, \infty}(\mathscr{M}, \mathscr{N})\right.$ and $Z_{*} \in L^{\infty}\left(T^{*} \mathscr{M} \times \mathbb{R}^{N}\right)$ with $\operatorname{div}_{\gamma} Z_{*} \in L^{\infty}\left(\mathscr{M}, \mathbb{R}^{N}\right)$ such that

$$
\begin{aligned}
\pi_{\mathbf{u}_{*}}\left(\operatorname{div}_{\gamma} Z_{*}\right) & =\mathbf{0}, \quad Z_{*} \in \frac{\mathrm{d} \mathbf{u}_{*}}{\left|\mathrm{~d} \mathbf{u}_{*}\right|_{\gamma}}, \\
\mathbf{u}\left(t_{k}, \cdot\right) & \rightarrow \mathbf{u}_{*} \operatorname{in} C(\mathscr{M}, \mathscr{N}) .
\end{aligned}
$$

As $\mathbf{u}$ is continuous and the sequence $\left(\mathbf{u}\left(t_{k}, \cdot\right)\right)$ converges to $\mathbf{u}_{*}$ in $C(\mathscr{M}, \mathscr{N}), \mathbf{u}_{*}$ and $\mathbf{u}_{0}$ are homotopic. Thus, we have solved the homotopy problem for (weakly) 1-harmonic maps assuming that $\mathscr{M}$ is orientable with Ric $_{\mathscr{M}} \geq 0$ and $K_{\mathscr{N}} \leq 0$.

The plan of the paper is the following one: Firstly, in Sect. 2, we prove Theorem 1. In Sect. 3, we obtain well-posedness of an approximating system to (2)-(4) and we obtain some a priori estimates (independent of the parameter of approximation) for their solutions. This permits us to prove Theorem 2, to which Sect. 4 is devoted. The asymptotic behaviour is treated in the next sections: in Sect. 5, we prove Theorem 3 while in Sect. 6, we treat the case of non-positive curvature; i.e., Theorem 4. Section 7 is devoted to the case where the domain is a compact Riemannian manifold, in which we prove Theorem 5. The last part of the paper is an appendix where some technical lemmata are stated and proven.

\section{Uniqueness}

In this section, we give the proof of Theorem 1.

Let $\left(\mathbf{u}^{1}, \mathrm{Z}^{1}\right),\left(\mathbf{u}^{2}, \mathrm{Z}^{2}\right)$ be two regular solutions to (2), (3). For $i=1,2$ there holds

$$
\mathbf{u}_{t}^{i}=\operatorname{div} Z^{i}+\mathscr{A}_{\mathbf{u}^{i}}\left(\mathbf{u}_{x^{j}}^{i}, \mathbf{Z}_{j}^{i}\right) \text {. }
$$

Here and in the rest of this section, $\mathbf{u}_{x j}^{i}$ and $\mathbf{Z}_{j}^{i}$ denote, respectively, the derivative of $\mathbf{u}^{i}$ in direction of $x^{j}$ and the $j$-th component of $Z^{i}, i=1,2, j=1, \ldots, m$. We calculate

$$
\begin{aligned}
\frac{1}{2} \frac{\mathrm{d}}{\mathrm{d} t} \int_{\Omega}\left|\mathbf{u}^{1}-\mathbf{u}^{2}\right|^{2}= & \int_{\Omega}\left(\mathbf{u}^{1}-\mathbf{u}^{2}\right) \cdot\left(\operatorname{div} Z^{1}-\operatorname{div} Z^{2}\right) \\
& +\int_{\Omega}\left(\mathbf{u}^{1}-\mathbf{u}^{2}\right) \cdot\left(\mathscr{A}_{\mathbf{u}^{1}}\left(\mathbf{u}_{x^{j}}^{1}, \mathbf{Z}_{j}^{1}\right)-\mathscr{A}_{\mathbf{u}^{2}}\left(\mathbf{u}_{x^{j}}^{2}, \mathbf{Z}_{j}^{2}\right)\right) .
\end{aligned}
$$

In the first term on the r.h.s. of (19) we integrate by parts, yielding

$$
\int_{\Omega}\left(\mathbf{u}^{1}-\mathbf{u}^{2}\right) \cdot\left(\operatorname{div} Z^{1}-\operatorname{div} Z^{2}\right)=-\int_{\Omega}\left(\left|\nabla \mathbf{u}^{1}\right|-\nabla \mathbf{u}^{1}: Z^{2}+\left|\nabla \mathbf{u}^{2}\right|-\nabla \mathbf{u}^{2}: Z^{1}\right)
$$

which is non-positive as $\left|Z^{i}\right| \leq 1, i=1,2$. Next, we note that for any $\mathbf{p}^{1}, \mathbf{p}^{2} \in \mathscr{N}$ contained in a fixed compact subset $K$ of $\mathscr{N}$ we have

$$
\left|\pi_{\mathbf{p}^{i}}^{\perp}\left(\mathbf{p}^{1}-\mathbf{p}^{2}\right)\right| \leq C_{1}(K)\left|\mathbf{p}^{1}-\mathbf{p}^{2}\right|^{2}
$$

for $i=1,2$. The exponent two on the right-hand side follows from the second-order Taylor expansion of $\pi \frac{\perp}{\mathbf{p}^{i}}\left(\mathbf{p}-\mathbf{p}^{i}\right)$ around $\mathbf{p}^{i}$ : indeed

$$
\mathbf{p}-\mathbf{p}^{i}=\exp _{\mathbf{p}^{i}}^{-1} \mathbf{p}+O\left(\left|\mathbf{p}-\mathbf{p}^{i}\right|^{2}\right)
$$


in a neighborhood $\mathscr{U} \subset \mathscr{N}$ of $\mathbf{p}^{i}$, where $\exp _{\mathbf{p}^{i}}^{-1}: \mathscr{U} \rightarrow T_{\mathbf{p}^{i}} \mathscr{N}$ is the logarithmic map of $\mathscr{N}$ at $\mathbf{p}^{i}$ (see e.g., [31, Lemma A.1]). Such exponent is crucial for the following Gronwall-type argument.

As $\mathbf{u}^{1}, \mathbf{u}^{2}$ are continuous on $[0, T] \times \bar{\Omega}$ (we assume without loss of generality that $T$ is finite), there is indeed a compact set $K=K\left(\mathbf{u}^{1}, \mathbf{u}^{2}\right)$ in $\mathscr{N}$ with $\mathbf{u}^{i}([0, T] \times \bar{\Omega}) \subset K$, $i=1,2$. Therefore, as $\mathscr{A}_{\mathbf{u}^{i}}$ is valued in $T_{\mathbf{u}^{i}} \mathscr{N}^{\perp}(i=1,2)$ there is a constant $C_{2}$ depending on $K$ and the norms of $\nabla \mathbf{u}^{1}, \nabla \mathbf{u}^{2}$ in $L^{\infty}(] 0, T\left[\times \Omega, \mathbb{R}^{N}\right)$ such that

$$
\frac{1}{2} \frac{\mathrm{d}}{\mathrm{d} t} \int_{\Omega}\left|\mathbf{u}^{1}-\mathbf{u}^{2}\right|^{2} \leq C_{2} \int_{\Omega}\left|\mathbf{u}^{1}-\mathbf{u}^{2}\right|^{2}
$$

for a.e., $t \in] 0, T\left[\right.$. Thus, if $\mathbf{u}^{1}(0, \cdot)=\mathbf{u}^{2}(0, \cdot)$, we have $\mathbf{u}^{1} \equiv \mathbf{u}^{2}$ due to Gronwall's lemma.

\section{The approximate system}

In this section, $\Omega \subset \mathbb{R}^{m}$ is assumed to be an open, bounded, smooth, convex domain and $0<\alpha<1$. Given $\varepsilon>0, T \in] 0, \infty]$ we consider the approximating system for $\mathbf{u}^{\varepsilon}:[0, T[\times \Omega \rightarrow \mathscr{N}:$

$$
\begin{aligned}
& \left.\mathbf{u}_{t}^{\varepsilon}=\pi_{\mathbf{u}^{\varepsilon}}\left(\operatorname{div} \frac{\nabla \mathbf{u}^{\varepsilon}}{\sqrt{\varepsilon^{2}+\left|\nabla \mathbf{u}^{\varepsilon}\right|^{2}}}\right) \text { in }\right] 0, T[\times \Omega, \\
& \left.\boldsymbol{v}^{\Omega} \cdot \nabla \mathbf{u}^{\varepsilon}=\mathbf{0} \text { in }\right] 0, T[\times \partial \Omega, \\
& \mathbf{u}^{\varepsilon}(0, \cdot)=\mathbf{u}_{0} .
\end{aligned}
$$

Further in this section, we will drop the index $\varepsilon$ and denote $Z=\frac{\nabla \mathbf{u}}{\sqrt{\varepsilon^{2}+|\nabla \mathbf{u}|^{2}}}$.

We will obtain solutions to (20)-(22) in parabolic Hölder spaces as defined in [26, Chapter I]. Let us introduce some necessary notation. Given numbers $k=0,1, \ldots, 0<\alpha<1$ and an interval $I$, we write $C^{\frac{k+\alpha}{2}, k+\alpha}\left(\Omega_{I}, \mathbb{R}^{N}\right)$ for the parabolic Hölder space on $\Omega_{I}=I \times \Omega$ of order $k+\alpha$. Similarly, we write $\mathbf{u} \in C_{l o c}^{\frac{k+\alpha}{2}, k+\alpha}\left(\bar{\Omega}_{I}, \mathbb{R}^{N}\right)$ if $\mathbf{u} \in C^{\frac{k+\alpha}{2}, k+\alpha}\left(\Omega_{K}, \mathbb{R}^{N}\right)$ for every interval $K$ compactly included in $I$.

\subsection{Uniform bounds}

In this subsection, we prove essential a priori estimates for $\mathbf{u} \in C_{l o c}^{\frac{3+\alpha}{2}, 3+\alpha}\left(\bar{\Omega}_{[0, T[}, \mathscr{N}\right)$ solving (20), (21) with a given $\varepsilon, T>0$. For brevity, we denote

$$
v=\left(|\nabla \mathbf{u}|^{2}+\varepsilon^{2}\right)^{\frac{1}{2}}, \quad v_{0}=\left(\left|\nabla \mathbf{u}_{0}\right|^{2}+\varepsilon^{2}\right)^{\frac{1}{2}} .
$$

The basic energy estimate reflects the gradient flow structure behind (20), (21).

Lemma 1 Let $\mathbf{u} \in C_{l o c}^{\frac{3+\alpha}{2}, 3+\alpha}\left(\bar{\Omega}_{[0, T[}, \mathscr{N}\right)$ satisfy (20), (21). Then

$$
\sup _{t \in[0, T[} \int_{\Omega} v(t, \cdot)+\int_{0}^{T} \int_{\Omega} \mathbf{u}_{t}^{2} \leq \int_{\Omega} v_{0} .
$$


Proof The estimate follows from the equality

$$
\frac{\mathrm{d}}{\mathrm{d} t} \int_{\Omega} v=\int_{\Omega} \mathrm{Z}: \nabla \mathbf{u}_{t}=-\int_{\Omega} \mathbf{u}_{t}^{2}
$$

which holds as $\mathbf{u}_{t}(t, \mathbf{x}) \in T_{\mathbf{u}(t, \mathbf{x})} \mathscr{N}$ for $\left.(t, \mathbf{x}) \in\right] 0, T[\times \Omega$.

In order to derive further uniform bounds, our main tool is the following version of Bochner's identity (see [29, Chapter 1] for the case of harmonic maps).

Lemma 2 Let $\mathbf{u} \in C_{l o c}^{\frac{3+\alpha}{2}, 3+\alpha}\left(\bar{\Omega}_{[0, T[}, \mathscr{N}\right)$ satisfy (20). Then, on $] 0, T[\times \Omega$,

$$
\frac{1}{2} \frac{\mathrm{d}}{\mathrm{d} t}|\nabla \mathbf{u}|^{2}=\left(\nabla \mathbf{u}: \nabla \mathbf{Z}_{i}\right)_{x^{i}}-\left(\pi_{\mathbf{u}} \nabla^{2} \mathbf{u}\right) \vdots \nabla Z+\mathbf{Z}_{i} \cdot \mathscr{R}_{\mathbf{u}}^{\mathscr{N}}\left(\mathbf{u}_{x^{i}}, \mathbf{u}_{x^{j}}\right) \mathbf{u}_{x^{j}}
$$

Proof Given $t \in] 0, T\left[, \mathbf{x} \in \Omega\right.$, we choose a local orthonormal frame $\left(\mathbf{N}^{k}\right)_{k=1, \ldots, N-n}$ on $\mathscr{N}$ around $\mathbf{u}(t, \mathbf{x})$. For any $\mathbf{p} \in \mathscr{N}$ close enough to $\mathbf{u}(t, \mathbf{x})$, we express using this frame

$$
\pi_{\mathbf{p}}^{\perp}=\mathbf{N}_{\mathbf{p}}^{k} \otimes \mathbf{N}_{\mathbf{p}}^{k}, \quad \mathscr{A}_{\mathbf{p}}(\mathbf{X}, \mathbf{Y})=\left(\mathbf{X} \cdot D_{\mathbf{p}} \mathbf{N}^{k} \mathbf{Y}\right) \mathbf{N}_{\mathbf{p}}^{k},
$$

where $\mathbf{X}, \mathbf{Y} \in T_{\mathbf{p}} \mathscr{N}, \mathbf{N}_{\mathbf{p}}^{k}$ denotes the value of $\mathbf{N}^{k}$ at $\mathbf{p}$ and $D_{\mathbf{p}} \mathbf{N}^{k}: T_{\mathbf{p}} \mathscr{N} \rightarrow \mathbb{R}^{N}$ is the tangent map to $\mathbf{N}^{k}$ at $\mathbf{p}$, that is $D_{\mathbf{w}} \mathbf{N}^{k} \mathbf{w}_{s}=\left(\mathbf{N}_{\mathbf{w}}^{k}\right)_{s}$ for any $C^{1}$ curve $s \mapsto \mathbf{w}(s) \in \mathscr{N}$. We recall that $\mathscr{A}_{\mathbf{p}}$ is symmetric and does not depend on the choice of $\left(\mathbf{N}^{k}\right)$ [38, Chapter 7], and by convention

$$
\mathbf{X} \otimes \mathbf{Y} \mathbf{u}=(\mathbf{Y} \cdot \mathbf{u}) \mathbf{X}, \quad(\mathbf{X} \otimes \mathbf{Y} \mathbf{u}) \cdot \mathbf{v}=(\mathbf{Y} \cdot \mathbf{u})(\mathbf{X} \cdot \mathbf{v})
$$

for any $\mathbf{X}, \mathbf{Y}, \mathbf{u}, \mathbf{v} \in \mathbb{R}^{N}$.

First, we calculate

$$
-\mathbf{N}_{\mathbf{u}}^{k} \otimes \mathbf{N}_{\mathbf{u}}^{k} \operatorname{div} \mathbf{Z}=-\mathbf{N}_{\mathbf{u}}^{k}(\underbrace{\mathbf{N}_{\mathbf{u}}^{k} \cdot \mathbf{Z}_{j}}_{=0})_{x^{j}}+\mathbf{N}_{\mathbf{u}}^{k}\left(\left(\mathbf{N}_{\mathbf{u}}^{k}\right)_{x^{j}} \cdot \mathbf{Z}_{j}\right)=\mathscr{A}_{\mathbf{u}}\left(\mathbf{u}_{x^{j}}, \mathbf{Z}_{j}\right)
$$

which allows us to rewrite (20) as

$$
\mathbf{u}_{t}=\operatorname{div} \mathbf{Z}+\mathscr{A}_{\mathbf{u}}\left(\mathbf{u}_{x^{j}}, \mathbf{Z}_{j}\right) .
$$

Using (27), we obtain

$$
\begin{aligned}
\frac{1}{2} \frac{\mathrm{d}}{\mathrm{d} t}|\nabla \mathbf{u}|^{2}= & \nabla \mathbf{u}: \nabla \operatorname{div} \mathbf{Z}+\nabla \mathbf{u}: \nabla \mathscr{A}_{\mathbf{u}}\left(\mathbf{u}_{x^{i}}, \mathbf{Z}_{i}\right) \\
= & \left(\nabla \mathbf{u}: \nabla \mathbf{Z}_{i}\right)_{x^{i}}-\nabla^{2} \mathbf{u} \vdots \nabla \mathbf{Z}+(\underbrace{\mathbf{u}_{x^{j}} \cdot \mathscr{A}_{\mathbf{u}}\left(\mathbf{u}_{x^{i}}, \mathbf{Z}_{i}\right)}_{=0})_{x^{j}} \\
& -\Delta \mathbf{u} \cdot \mathscr{A}_{\mathbf{u}}\left(\mathbf{u}_{x^{i}}, \mathbf{Z}_{i}\right)
\end{aligned}
$$

where in the last line we used that $\mathscr{A}_{\mathbf{u}}$ is orthogonal to $\mathbf{u}_{x^{j}} \in T_{\mathbf{u}} \mathscr{N}$.

Next we perform the following calculations:

$$
\begin{aligned}
\left(\pi_{\mathbf{u}}^{\perp} \nabla^{2} \mathbf{u}\right) \vdots \nabla \mathbf{Z} \stackrel{(25)}{=} & \left(\mathbf{N}_{\mathbf{u}}^{k} \otimes \mathbf{N}_{\mathbf{u}}^{k} \nabla^{2} \mathbf{u}\right) \vdots \nabla \mathbf{Z}=\left(\mathbf{N}_{\mathbf{u}}^{k} \otimes \mathbf{N}_{\mathbf{u}}^{k} \mathbf{u}_{x^{i} x^{j}}\right) \cdot \mathbf{Z}_{i, x^{j}} \\
& \stackrel{(26)_{2}}{=} \mathbf{N}_{\mathbf{u}}^{k} \cdot \mathbf{u}_{x^{i} x^{j}} \mathbf{N}_{\mathbf{u}}^{k} \cdot \mathbf{Z}_{i, x^{j}} \\
= & \left.\left.(\underbrace{\left(\mathbf{N}_{\mathbf{u}}^{k} \cdot \mathbf{u}_{x^{i}}\right.}_{=0})_{x^{j}}-\left(\mathbf{N}_{\mathbf{u}}^{k}\right)_{x^{j}} \cdot \mathbf{u}_{x^{i}}\right)(\underbrace{\left(\mathbf{N}_{\mathbf{u}}^{k} \cdot \mathbf{Z}_{i}\right.}_{=0})_{x^{j}}-\left(\mathbf{N}_{\mathbf{u}}^{k}\right)_{x^{j}} \cdot \mathbf{Z}_{i}\right) \\
= & \mathscr{A}_{\mathbf{u}}\left(\mathbf{u}_{x^{i}}, \mathbf{u}_{x^{j}}\right) \cdot \mathscr{A}_{\mathbf{u}}\left(\mathbf{u}_{x^{j}}, \mathbf{Z}_{i}\right)
\end{aligned}
$$


and similarly

$$
\left.\left.\pi_{\mathbf{u}}^{\perp} \Delta \mathbf{u} \stackrel{(26}{=}\right)_{1}\left(\mathbf{N}_{\mathbf{u}}^{k} \cdot \mathbf{u}_{x^{j} x^{j}}\right) \mathbf{N}_{\mathbf{u}}^{k}=(\underbrace{\left(\mathbf{N}_{\mathbf{u}}^{k} \cdot \mathbf{u}_{x^{j}}\right.}_{=0})_{x^{j}}-\left(\mathbf{N}_{\mathbf{u}}^{k}\right)_{x^{j}} \cdot \mathbf{u}_{x^{j}}\right) \mathbf{N}_{\mathbf{u}}^{k}=-\mathscr{A}_{\mathbf{u}}\left(\mathbf{u}_{x^{j}}, \mathbf{u}_{x^{j}}\right),
$$

so that

$$
\Delta \mathbf{u} \cdot \mathscr{A}_{\mathbf{u}}\left(\mathbf{u}_{x^{i}}, \mathbf{Z}_{i}\right)=\pi_{\mathbf{u}}^{\perp} \Delta \mathbf{u} \cdot \mathscr{A}_{\mathbf{u}}\left(\mathbf{u}_{x^{i}}, \mathbf{Z}_{i}\right)=-\mathscr{A}_{\mathbf{u}}\left(\mathbf{u}_{x^{j}}, \mathbf{u}_{x^{j}}\right) \cdot \mathscr{A}_{\mathbf{u}}\left(\mathbf{u}_{x^{i}}, \mathbf{Z}_{i}\right) .
$$

Hence, (28) may be rewritten as

$$
\begin{aligned}
\frac{1}{2} \frac{\mathrm{d}}{\mathrm{d} t}|\nabla \mathbf{u}|^{2}= & \left(\nabla \mathbf{u}: \nabla \mathbf{Z}_{i}\right)_{x^{i}}-\left(\pi_{\mathbf{u}} \nabla^{2} \mathbf{u}\right) \vdots \nabla \mathbf{Z} \\
& -\mathscr{A}_{\mathbf{u}}\left(\mathbf{u}_{x^{i}}, \mathbf{u}_{x^{j}}\right) \cdot \mathscr{A}_{\mathbf{u}}\left(\mathbf{u}_{x^{j}}, \mathbf{Z}_{i}\right)+\mathscr{A}_{\mathbf{u}}\left(\mathbf{u}_{x^{j}}, \mathbf{u}_{x^{j}}\right) \cdot \mathscr{A}_{\mathbf{u}}\left(\mathbf{u}_{x^{i}}, \mathbf{Z}_{i}\right) .
\end{aligned}
$$

Finally, we recall the Gauss-Codazzi equation

$$
\mathbf{W} \cdot \mathscr{R}_{\mathbf{p}}^{\mathscr{N}}(\mathbf{X}, \mathbf{Y}) \mathbf{Z}=\mathscr{A}_{\mathbf{p}}(\mathbf{Y}, \mathbf{Z}) \cdot \mathscr{A}_{\mathbf{p}}(\mathbf{X}, \mathbf{W})-\mathscr{A}_{\mathbf{p}}(\mathbf{X}, \mathbf{Z}) \cdot \mathscr{A}_{\mathbf{p}}(\mathbf{Y}, \mathbf{W})
$$

for any quadruple of vectors $\mathbf{X}, \mathbf{Y}, \mathbf{Z}, \mathbf{W} \in T_{\mathbf{p}} \mathscr{N}, p \in \mathscr{N}$, which finishes the proof.

We are now ready to derive uniform Lipschitz bounds.

Lemma 3 Let $\mathbf{u} \in C_{l o c}^{\frac{3+\alpha}{2}, 3+\alpha}\left(\bar{\Omega}_{[0, T[}, \mathscr{N}\right)$ satisfy (20)-(22).

(i) If $\left.K_{\mathscr{N}} \in\right] 0, \infty[$, then

$$
\|v(t, \cdot)\|_{L^{\infty}} \leq \frac{\left\|v_{0}\right\|_{L^{\infty}}}{1-t K_{\mathscr{N}}\left\|v_{0}\right\|_{L^{\infty}}}
$$

for $t \in] 0, \min \left(T_{\dagger}, T\right)\left[\right.$, where $T_{\dagger}:=\left(K_{\mathscr{N}}\left\|v_{0}\right\|_{L^{\infty}}\right)^{-1}$.

(ii) If $K_{\mathscr{N}} \leq 0$, then for $0<t<T<T_{\dagger}:=+\infty$ there holds

$$
\|v(t, \cdot)\|_{L^{\infty}} \leq\left\|v_{0}\right\|_{L^{\infty}} .
$$

Proof Given a finite $p \geq 1$, using (24) and integrating by parts, we calculate

$$
\begin{aligned}
\frac{1}{p} \frac{\mathrm{d}}{\mathrm{d} t} \int_{\Omega} v^{p}= & \int_{\Omega} v^{p-2} \nabla \mathbf{u}: \nabla \mathbf{u}_{t} \\
= & -\int_{\Omega} v^{p-2}\left(\pi_{\mathbf{u}} \nabla^{2} \mathbf{u}\right): \nabla \mathbf{Z}-(p-2) \int_{\Omega} v^{p-4} \nabla \mathbf{u}: \nabla^{2} \mathbf{u} \cdot \nabla \mathbf{Z}: \nabla \mathbf{u} \\
& +\int_{\partial \Omega} v^{p-2}\left(\nabla \mathbf{u}: \nabla \mathbf{Z}_{i}\right)\left(v^{\Omega}\right)^{i}+\int_{\Omega} v^{p-3} \mathbf{u}_{x^{i}} \cdot \mathscr{R}_{\mathbf{u}}^{\mathcal{N}}\left(\mathbf{u}_{x^{i}}, \mathbf{u}_{x^{j}}\right) \mathbf{u}_{x^{j}} .
\end{aligned}
$$

We have

$$
Z_{j, x^{k}}^{i}=v^{-1}\left(u_{x^{j} x^{k}}^{i}-Z_{j}^{i}\left(\nabla \mathbf{u}_{x^{k}}: Z\right)\right)
$$

and

$$
\nabla \mathbf{Z}_{j}: \nabla \mathbf{u}=v^{-1}\left(\nabla \mathbf{u}: \nabla \mathbf{u}_{x^{j}}-Z_{j}^{i} Z_{k}^{i} \nabla \mathbf{u}_{x^{k}}: \nabla \mathbf{u}\right)
$$

for $i=1, \ldots, N$ and $j, k=1, \ldots, m$. Thus, we can rewrite

$$
\nabla \mathbf{u}: \nabla^{2} \mathbf{u} \cdot \nabla \mathbf{Z}: \nabla \mathbf{u}=v^{-1} \nabla \mathbf{u}: \nabla \mathbf{u}_{x^{j}}\left(I_{j k}^{m}-Z_{j}^{i} Z_{k}^{i}\right) \nabla \mathbf{u}_{x^{k}}: \nabla \mathbf{u}
$$


(we use the notation $\mathrm{I}^{l}=\left(I_{j k}^{l}: j, k=1, \ldots, l\right)$ for the $l$-dimensional identity matrix). On the other hand,

$$
\left(\pi_{\mathbf{u}} \nabla^{2} \mathbf{u}\right) \vdots \nabla \mathbf{Z}=v^{-1}\left(\pi_{\mathbf{u}} \nabla \mathbf{u}_{x^{j}}\right):\left(\mathrm{I}^{m} \otimes \mathrm{I}^{N}-\mathbf{Z} \otimes \mathbf{Z}\right): \nabla \mathbf{u}_{x^{j}} .
$$

From (32), (33) and the fact that $|Z| \leq 1$, it is clear that, provided $p \geq 2$, the first two terms on the r.h.s. of (31) are non-positive. To treat the remaining boundary term, we extend $\boldsymbol{v}^{\Omega}$ to a normal tubular neighbourhood of $\partial \Omega$ in such a way that it is constant in the fibers, and calculate (at points in $\partial \Omega$ )

$$
\begin{aligned}
\left(\nabla \mathbf{u}: \nabla \mathbf{Z}_{i}\right)\left(\boldsymbol{v}^{\Omega}\right)^{i} & =\nabla u^{j} \cdot \nabla\left(Z_{i}^{j}\left(\boldsymbol{v}^{\Omega}\right)^{i}\right)-\nabla u^{j} \cdot \nabla\left(\boldsymbol{v}^{\Omega}\right)^{i} Z_{i}^{j} \\
& =-v^{-1} \boldsymbol{v}^{\Omega} \cdot \mathscr{A}^{\partial \Omega}\left(\nabla u^{i}, \nabla u^{i}\right) .
\end{aligned}
$$

The term $\nabla u^{j} \cdot \nabla\left(Z_{i}^{j}\left(\boldsymbol{v}^{\Omega}\right)^{i}\right)$ vanishes because, due to (21), $\nabla u^{j} \in T_{\mathbf{x}} \partial \Omega$ and $\nabla\left(Z_{i}^{j}\left(\boldsymbol{v}^{\Omega}\right)^{i}\right) \in$ $\left(T_{\mathbf{x}} \partial \Omega\right)^{\perp}$ for $j=1, \ldots N$. By $\mathscr{A}^{\partial \Omega}$ we denoted the second fundamental form of hypersurface $\partial \Omega$,

$$
\mathscr{A}_{\mathbf{X}}^{\partial \Omega}(\mathbf{X}, \mathbf{Y})=\left(\mathbf{X} \cdot D_{\mathbf{x}} \boldsymbol{v}^{\Omega} \mathbf{Y}\right) \boldsymbol{v}_{\mathbf{x}}^{\Omega}
$$

for $\mathbf{x} \in \partial \Omega$, where index $\mathbf{x}$ on $\mathscr{A}^{\partial \Omega}, \boldsymbol{v}^{\Omega}$ denotes evaluation at $\mathbf{x}$ and $D_{\mathbf{x}} \boldsymbol{v}^{\Omega}: T_{\mathbf{x}} \partial \Omega \rightarrow \mathbb{R}^{m}$ is the tangent map of $\boldsymbol{v}^{\Omega}$ at $\mathbf{x}$ (see the remark after (25)). As $\Omega$ is convex, $\boldsymbol{v}^{\Omega} \cdot \mathscr{A}^{\partial \Omega}$ is non-negative. This ends the proof of (30) in the case $K_{\mathscr{N}} \leq 0$.

Now, assume that $\left.K_{\mathscr{N}} \in\right] 0, \infty[$. By virtue of the previous calculations and (9), we have

$$
\frac{\mathrm{d}}{\mathrm{d} t}\left(\int_{\Omega} v^{p}\right)^{\frac{1}{p}} \leq\left(\int_{\Omega} v^{p}\right)^{\frac{1}{p}-1} K_{\mathscr{N}} \int_{\Omega} v^{p+1} \leq K_{\mathscr{N}}\left(\int_{\Omega} v^{p}\right)^{\frac{1}{p}}\|v\|_{L^{\infty}} .
$$

Passing to the limit $p \rightarrow \infty$ we obtain, at least in a weak sense,

$$
\frac{\mathrm{d}}{\mathrm{d} t}\|v\|_{L^{\infty}} \leq K_{\mathscr{N}}\|v\|_{L^{\infty}}^{2}
$$

which implies (29).

\subsection{Existence for the approximate system}

In order to prove existence of solutions to the approximate system we proceed similarly as in [23, Section 3.]. The assumption that the embedding of $\mathscr{N}$ into $\mathbb{R}^{N}$ is closed enables us to construct a metric $h$ on $\mathbb{R}^{N}$ such that $(\mathscr{N}, g)$ is a totally geodesic Riemannian submanifold of $\left(\mathbb{R}^{N}, h\right)$ (see Lemma A.1 in the appendix), i.e.,

- the restriction of $h$ to $T \mathscr{N}$ coincides with $g$, that is $\left.h_{\mathbf{p}}\right|_{T_{\mathbf{p}} \mathscr{N} \times T_{\mathbf{p}} \mathscr{N}} \equiv g_{\mathbf{p}}$ for $\mathbf{p} \in \mathscr{N}$,

- there is a tubular neighborhood $\mathscr{T}$ of $\mathscr{N}$ in $\mathbb{R}^{N}$ such that the involution $\tau: \mathscr{T} \rightarrow \mathscr{T}$ given by multiplication by -1 in the fibers of $\mathscr{T}$ is an isometry.

The gradient flow of the unconstrained functional $\int_{\Omega}|\nabla \mathbf{u}|_{h}$ defined for any regular enough function $\mathbf{u}: \Omega \rightarrow \mathbb{R}^{N}$ is expressed by the system

$$
\begin{aligned}
& u_{t}^{i}=\operatorname{div} \frac{\nabla u^{i}}{\sqrt{\varepsilon^{2}+|\nabla \mathbf{u}|_{h}^{2}}}+\frac{1}{\sqrt{\varepsilon^{2}+|\nabla \mathbf{u}|_{h}^{2}}} \Gamma_{j k}^{i}(\mathbf{u}) u_{x^{l}}^{j} u_{x^{l}}^{k}, \\
& \boldsymbol{v}^{\Omega} \cdot \nabla u^{i}=0
\end{aligned}
$$

where $i=1, \ldots, N$ and $\Gamma_{j k}^{i}$ are the Christoffel symbols of $\left(\mathbb{R}^{N}, h\right)$. As $h$ restricted to $T \mathscr{N}$ coincides with $g$, the system (35), (36) is identical to (20), (36) as long as the range of $\mathbf{u}$ is 
contained in $\mathscr{N}$. In order for $C_{l o c}^{\frac{3+\alpha}{2}, 3+\alpha}\left(\Omega_{[0, T[}, \mathscr{N}\right)$ solutions to the system (35), (36) with initial datum $\mathbf{u}_{0}$ to exist, the following compatibility conditions

$$
\begin{aligned}
& \boldsymbol{v}^{\Omega} \cdot \nabla u_{0}^{i}=0 \\
& \boldsymbol{v}^{\Omega} \cdot \nabla\left(\operatorname{div} \frac{\nabla u_{0}^{i}}{\sqrt{\varepsilon^{2}+\left|\nabla \mathbf{u}_{0}\right|_{h}^{2}}}+\frac{1}{\sqrt{\varepsilon^{2}+\left|\nabla \mathbf{u}_{0}\right|_{h}^{2}}} \Gamma_{j k}^{i}\left(\mathbf{u}_{0}\right) u_{0, x^{l}}^{j} u_{0, x^{l}}^{k}\right)=0
\end{aligned}
$$

on $\partial \Omega$ for $i=1, \ldots, N$ need to be satisfied.

Proposition 1 Suppose that $K_{\mathscr{N}}<\infty$ and $\left.\alpha \in\right] 0,1\left[\right.$. Let $\mathbf{u}_{0} \in C^{3+\alpha}(\Omega, \mathscr{N})$ satisfy (37), (38). Then for any $\varepsilon>0$ the system (20)-(22) has a unique solution

$$
\mathbf{u} \in C_{l o c}^{\frac{3+\alpha}{2}, 3+\alpha}\left(\bar{\Omega}_{\left[0, T_{\dagger}[\right.}, \mathscr{N}\right)
$$

where $\left.\left.T_{\dagger}=T_{\dagger}\left(\left\|\nabla \mathbf{u}_{0}\right\|_{L^{\infty}}, K_{\mathscr{N}}\right) \in\right] 0, \infty\right]$ is defined in Lemma 3.

Note that $T_{\dagger}$ in Proposition 1 does not depend on $\varepsilon$.

The expressions on the right hand side of (35) make sense without assuming a priori that the range of $\mathbf{u}$ is contained in $\mathscr{N}$. This fact enables us to obtain a local-in-time solution using known results for parabolic systems. For that purpose, the authors in [23] or in [12] combine a general existence result from [30] with sectoriality estimates from [44]. On the other hand, in [32] the author employs estimates from [37] and [33]. However, both [44] and [37] can only be applied to the system with Dirichlet boundary condition, or to the case with no boundary. As we are dealing with homogeneous Neumann boundary condition, we appeal instead to a result of Acquistapace and Terreni [1, Theorem 1.1.] for quasilinear systems with general boundary conditions.

To justify its applicability to our problem, let us briefly check the assumptions. We can rewrite the divergence part of the right hand side of (35) as $\mathrm{A}_{k l}(\nabla \mathbf{u}) \mathbf{u}_{x^{k} x^{l}}$, where $\mathrm{A}_{k l}: \mathbb{R}^{N} \rightarrow$ $\mathbb{R}^{N}$ is given by

$$
\mathrm{A}_{k l}(\mathrm{P})=\frac{1}{\sqrt{\varepsilon^{2}+|\mathrm{P}|_{h}^{2}}}\left(I_{k l}^{m} I^{N}-\frac{\mathbf{P}_{k}}{\sqrt{\varepsilon^{2}+|\mathrm{P}|_{h}^{2}}} \otimes \frac{\mathbf{P}_{l}}{\sqrt{\varepsilon^{2}+|\mathrm{P}|_{h}^{2}}}\right),
$$

for $k, l=1, \ldots, m$ with $\mathrm{P}=\left(\mathbf{P}_{1}, \ldots, \mathbf{P}_{m}\right) .\left(\mathrm{A}_{k l}\right)$ defines a locally uniformly strongly elliptic operator (see e.g., [2]) and therefore satisfies assumption (0.2) from [1]. It is easy to check that (36) satisfies the complementarity condition (0.3) from [1], and that the system satisfies regularity condition $(0.4)$ from [1].

Thus, as $\mathbf{u}_{0} \in C^{2+\alpha}(\Omega, \mathscr{N})$ satisfies compatibility condition (37), we obtain for any $p>m$ the existence of unique solution to (21), (35) with initial datum $\mathbf{u}_{0}$ in $C^{1+\frac{\alpha}{2}}\left(\left[0, T_{0}\left[, L^{p}\left(\Omega, \mathbb{R}^{N}\right)\right) \cap C^{\frac{\alpha}{2}}\left(\left[0, T_{0}\left[, W^{2, p}\left(\Omega, \mathbb{R}^{N}\right)\right)\right.\right.\right.\right.$ for some $T_{0}>0$. We choose $p$ so that $W^{2, p}(\Omega) \subset C^{1, \alpha}(\Omega)$. Then, we can treat the system (21), (35) as a linear system with $C^{\frac{\alpha}{2}, \alpha}$ coefficients and apply [26, Theorem VII.10.1] to obtain $\mathbf{u} \in C^{1+\frac{\alpha}{2}, 2+\alpha}\left(\Omega_{\left[0, T_{0}[\right.}\right)$. As long as $\mathbf{u}(t, \cdot) \in C^{2+\alpha}\left(\Omega, \mathbb{R}^{N}\right)$, we can extend the solution via Acquistapace-Terreni theorem. Therefore, there exists a maximal time $T_{*} \leq \infty$ such that

- u exists in $C_{l o c}^{1+\frac{\alpha}{2}, 2+\alpha}\left(\bar{\Omega}_{\left[0, T_{*}[\right.}, \mathbb{R}^{N}\right)$,

- the norm of $\mathbf{u}$ in $C^{1+\frac{\alpha}{2}, 2+\alpha}\left(\Omega_{[0, t[}, \mathbb{R}^{N}\right)$ blows up as $t \rightarrow T_{*}^{-}$if $T_{*}<\infty$.

Since $\mathbf{u} \in C_{l o c}^{1+\frac{\alpha}{2}, 2+\alpha}\left(\bar{\Omega}_{\left[0, T_{*}[\right.}, \mathbb{R}^{N}\right)$, the coefficients of (35), seen as a linear equation, belong to $C_{l o c}^{\frac{1+\alpha}{2}, 1+\alpha}\left(\bar{\Omega}_{\left[0, T_{*}\right.}[)\right.$. Therefore, provided $\mathbf{u}_{0} \in C^{3+\alpha}\left(\Omega, \mathbb{R}^{N}\right)$ and the additional compat- 
ibility condition (38) is satisfied, we may appeal once more to [26, Theorem VII.10.1] and conclude that $\mathbf{u} \in C_{l o c}^{\frac{3+\alpha}{2}, 3+\alpha}\left(\bar{\Omega}_{\left[0, T_{*}\right.}, \mathbb{R}^{N}\right)$.

We now argue that $\mathbf{u}(t, \Omega) \subset \mathscr{N}$ for all $t \in\left[0, T_{*}\right.$ [. Suppose, to the contrary, that there is $t \in] 0, T_{*}\left[\right.$ with $\mathbf{u}(t, \Omega) \not \subset \mathscr{N}$. Let $T_{\mathscr{N}}$ be the first time instance such that $\mathbf{u}(t, \Omega) \not \subset \mathscr{N}$ for $T_{\mathscr{N}}<t<T_{\mathscr{N}}+\delta$ with some $\delta>0$. Possibly diminishing $\delta$ we can assume that $\mathbf{u}(t, \Omega) \subset \mathscr{T}$ for $t \in\left[0, T_{\mathscr{N}}+\delta[\right.$. Then $\tau \circ \mathbf{u}$ is a solution to (35) different to $\mathbf{u}$ with the same initial and boundary conditions, thus violating uniqueness. Therefore, $\mathbf{u}(t, \Omega) \subset \mathscr{N}$ for all $t \in\left[0, T_{*}[\right.$.

It remains to show that $T_{*} \geq T_{\dagger}$, where $T_{\dagger}$ is defined in Lemma 3. Suppose that $T_{*}<T_{\dagger}$. Lemma 3 yields

$$
\sup _{t \in\left[0, T_{*}[\right.}\|\nabla \mathbf{u}(t, \cdot)\|_{L^{\infty}(\Omega)}<\infty .
$$

Let now $q>\frac{m+2}{1-\alpha}$. According to [26, Theorem VII.10.4 and Lemma II.3.3], there holds $\mathbf{u} \in W^{1, q}(] 0, T_{*}\left[, L^{q}\left(\Omega, \mathbb{R}^{N}\right)\right) \cap L^{q}(] 0, T_{*}\left[, W^{2, q}\left(\Omega, \mathbb{R}^{N}\right)\right)$ and consequently $\nabla \mathbf{u} \in$ $C^{\frac{\alpha}{2}, \alpha}\left(\Omega_{\left[0, T_{*}[\right.}, \mathbb{R}^{m \cdot N}\right)$. Now, [26, Theorem VII.10.1] yields $\mathbf{u} \in C^{1+\frac{\alpha}{2}, 2+\alpha}\left(\Omega_{\left[0, T_{*}[\right.}, \mathbb{R}^{N}\right)$, a contradiction.

\section{Local existence}

In this section we prove Theorem 2 .

Step 1 We assume that $\Omega$ is smooth and the initial datum $\mathbf{u}_{0} \in C^{3+\alpha}(\Omega)$ satisfies the compatibility conditions (37), (38). We want to pass to the limit $\varepsilon \rightarrow 0^{+}$in (20)-(22). Owing to Lemmata 1 and 3, we have uniform bounds on $\mathbf{u}_{t}^{\varepsilon}$ in $L^{2}(] 0, T[\times \Omega)$ and on $\nabla \mathbf{u}^{\varepsilon}$ in $L^{\infty}(] 0, T[\times \Omega)$ for any $T<T_{\dagger}$. Consequently, we also have a uniform bound on $\mathbf{u}^{\varepsilon}$ in $C^{\frac{1}{n+1}}(] 0, T[\times \Omega)[22]$. All these imply that we can extract a sequence $\left(\mathbf{u}_{k}\right)=\left(\mathbf{u}^{\varepsilon_{k}}\right)$ from $\left(\mathbf{u}^{\varepsilon}\right)$ such that

$$
\mathbf{u}_{k} \rightarrow \mathbf{u} \quad \text { in } C([0, T] \times \bar{\Omega}), \quad \nabla \mathbf{u}_{k} \rightarrow \nabla \mathbf{u} \quad \text { in } \quad L^{2}(] 0, T[\times \Omega) .
$$

Due to definition of $Z^{\varepsilon}$, we have $\left\|Z^{\varepsilon}\right\|_{L^{\infty}} \leq 1$, hence

$$
\left.\mathrm{Z}_{k} \stackrel{*}{\rightarrow} \mathrm{Z} \text { in } L^{\infty}(] 0, T[\times \Omega) \text { with }|\mathrm{Z}| \leq 1 \text { a. e. in }\right] 0, T[\times \Omega
$$

for a sequence $\left(Z_{k}\right)=\left(Z^{\varepsilon_{k}}\right)$. Furthermore, by virtue of the strong convergence of $\mathbf{u}_{k}$,

$$
0=\pi_{\mathbf{u}_{k}}^{\perp} \mathrm{Z}_{k} \stackrel{*}{\rightarrow} \pi_{\mathbf{u}}^{\perp} \mathrm{Z} \quad \text { in } L^{\infty}(] 0, T[\times \Omega) .
$$

Next, note that due to the Hölder bound, the family $\mathbf{u}^{\varepsilon}$ is contained in a compact subset of $\mathscr{N}$. Rewriting (20) as

$$
\mathbf{u}_{t}^{\varepsilon}=\operatorname{div} \mathbf{Z}^{\varepsilon}+\mathscr{A}_{\mathbf{u}^{\varepsilon}}\left(\mathbf{u}_{x^{i}}^{\varepsilon}, \mathbf{Z}_{i}^{\varepsilon}\right),
$$

we deduce a uniform bound on $\operatorname{div} Z^{\varepsilon}$ in $L^{2}(] 0, T[\times \Omega)$. By a standard div-curl reasoning,

$$
\nabla \mathbf{u}_{k}: Z_{k} \rightarrow \nabla \mathbf{u}: \mathbf{Z} \text { in } L^{2}(] 0, T[\times \Omega) .
$$

A simple calculation shows that

$$
\nabla \mathbf{u}^{\varepsilon}: Z^{\varepsilon}=\frac{\left|\nabla \mathbf{u}^{\varepsilon}\right|^{2}}{\sqrt{\varepsilon^{2}+\left|\nabla \mathbf{u}^{\varepsilon}\right|^{2}}} \geq\left|\nabla \mathbf{u}^{\varepsilon}\right|-\varepsilon
$$


Hence, by lower semicontinuity of $|\cdot|$ with respect to weak convergence, we get

$$
\nabla \mathbf{u}: Z \geq|\nabla \mathbf{u}|
$$

Collecting (40), (41), (43), (45) we obtain that $\nabla \mathbf{u}$ and $Z$ satisfy (5). Boundedness of $\operatorname{div} Z^{\varepsilon}$ in $L^{2}(] 0, T[\times \Omega)$ together with strong convergence of $\mathbf{u}_{k}$ is enough to pass to the limit in (20), (21), obtaining that $\nabla \mathbf{u}$ and Z satisfy (6), (7).

Step 2 Now, we relax the regularity assumption on the initial datum to $\mathbf{u}_{0} \in W^{1, \infty}(\Omega, \mathscr{N})$. Take a sequence $\left(\mathbf{u}_{0, j}\right) \subset C^{\infty}(\bar{\Omega}, \mathscr{N})$ such that $\mathbf{u}_{0, j}$ converges uniformly to $\mathbf{u}_{0}$, satisfies the compatibility conditions (37), (38) and

$$
\left\|\nabla \mathbf{u}_{0, j}\right\|_{L^{\infty}} \rightarrow\left\|\nabla \mathbf{u}_{0}\right\|_{L^{\infty}}
$$

Such a sequence is produced in Lemma A.2. By the previous step, there exists a regular solution $\left(\mathbf{u}_{j}, Z_{j}\right)$ to (2), (3) with initial datum $\mathbf{u}_{0, j}$. Recall that due to the form of estimates in Lemmata 1 and 3 the norms of $\mathbf{u}_{j, t}$ in $L^{2}(] 0, T\left[\times \Omega, \mathbb{R}^{N}\right)$ and of $\nabla \mathbf{u}_{j}$ in $L^{\infty}(] 0, T\left[\times \Omega, \mathbb{R}^{m \cdot N}\right)$ are controlled by $\left\|\nabla \mathbf{u}_{0, j}\right\|_{L^{\infty}}$. By virtue of (46), this control is uniform with respect to $j$. Hence, we can extract a subsequence converging to a regular solution to (2)-(4) following the same argument as in the previous step, with $\left(\mathbf{u}^{\varepsilon}, Z^{\varepsilon}\right)$ replaced by $\left(\mathbf{u}_{j}, Z_{j}\right)$, except that now we have $\nabla \mathbf{u}_{j}: Z_{j}=\left|\nabla \mathbf{u}_{j}\right|$ instead of (44).

Step 3 Next, we lift the smoothness assumption on the domain. A convex domain $\Omega$ can be approximated with respect to the Hausdorff distance by smooth convex domains $\Omega_{k} \subset$ $\Omega, k=1,2, \ldots$ For a proof of this result using the signed distance function of $\Omega$, see Lemma A. 3 in the appendix. The reasoning in the previous paragraph yields a sequence of pairs $\left(\mathbf{u}_{k}, Z_{k}\right.$ ), with $k$-th one satisfying (5)-(7) in $] 0, T\left[\times \Omega_{k}\right.$ with initial datum $\left.\mathbf{u}_{0}\right|_{\Omega_{k}}$. The estimates provided by Lemmata 1 and 3 are uniform with respect to $k$. Hence, we can use them as before together with a diagonal argument to extract subsequences of $\left(\mathbf{u}_{k}\right),\left(Z_{k}\right)$ that converge on compact subsets of $[0, T[\times \Omega$ to a regular solution (u, Z) to (2), (4) in $] 0, T[\times \Omega$.

Finally, we argue that the boundary condition (7) is satisfied. Let us fix $\varphi \in C^{1}(] 0, T[\times \bar{\Omega})$. We have

$$
\begin{aligned}
& \int_{0}^{T} \int_{\partial \Omega} \varphi \boldsymbol{v}^{\Omega} \cdot \mathrm{Z}=\int_{0}^{T} \int_{\Omega} \varphi \operatorname{div} \mathrm{Z}+\nabla \varphi \cdot \mathrm{Z}, \\
& 0=\int_{0}^{T} \int_{\partial \Omega_{k}} \varphi \boldsymbol{v}^{\Omega_{k}} \cdot \mathrm{Z}_{k}=\int_{0}^{T} \int_{\Omega_{k}} \varphi \operatorname{div} \mathrm{Z}_{k}+\nabla \varphi \cdot \mathrm{Z}_{k} .
\end{aligned}
$$

Let us denote $f=\varphi \operatorname{div} \mathbf{Z}+\nabla \varphi \cdot \mathbf{Z}, f_{k}=\varphi \operatorname{div} \mathbf{Z}_{k}+\nabla \varphi \cdot \mathbf{Z}_{k}$. By virtue of Hausdorff convergence, for a given $\varepsilon>0$, we are allowed to choose $K \subset \Omega$ and $k_{0}$ so that $\mid] 0, T\left[\times(\Omega \backslash K) \mid \leq \varepsilon^{2}\right.$ and $K \subset \Omega_{k}$ for $k \geq k_{0}$. Recalling (42), we note that $\left\|f_{k}\right\|_{L^{2}(] 0, T\left[\times \Omega_{k}\right)}$ is controlled in terms of norms $\left\|\mathbf{u}_{k, t}\right\|_{L^{2}(] 0, T\left[\times \Omega_{k}\right)}$ and $\left\|\nabla \mathbf{u}_{k}\right\|_{L^{\infty}(] 0, T\left[\times \Omega_{k}\right)}$ and hence is uniformly bounded. We can assume that $\left(\left.f_{k}\right|_{K}\right)_{k=k_{0}}^{\infty}$ converges weakly to $\left.f\right|_{K}$ in $L^{2}(] 0, T[\times K)$. Thus, we can choose $k \geq k_{0}$ large enough so that $\left|\int_{0}^{T} \int_{K} f-f_{k}\right| \leq \varepsilon$. We estimate

$$
\begin{aligned}
\left|\int_{0}^{T} \int_{\partial \Omega} \varphi \boldsymbol{v}^{\Omega} \cdot \mathrm{z}\right| & \leq\left|\int_{0}^{T} \int_{K} f-f_{k}\right|+\left|\int_{0}^{T} \int_{\Omega \backslash K} f\right|+\left|\int_{0}^{T} \int_{\Omega_{k} \backslash K} f_{k}\right| \\
& \leq\left(1+\|f\|_{L^{2}(] 0, T[\times \Omega)}+\left\|f_{k}\right\|_{L^{2}(] 0, T\left[\times \Omega_{k}\right)}\right) \varepsilon .
\end{aligned}
$$

As $\varepsilon$ and $\varphi$ are arbitrary, we are done. 


\section{Finite extinction time}

In order to prove Theorem 3 we will work directly with regular solutions to (2)-(4) in local coordinates $\mathbf{p} \mapsto\left(p^{1}, \ldots, p^{n}\right)$ on $\mathscr{N}$, in which (6) is expressed [11] as

$$
u_{t}^{i}=\operatorname{div} Z^{i}+\Gamma_{j k}^{i}(\mathbf{u}) u_{x^{l}}^{j} Z_{l}^{k}, \quad i=1, \ldots, n,
$$

where $\Gamma_{j k}^{i}$ are the Christoffel symbols of the chosen coordinate system. For $\mathbf{p}_{0} \in \mathscr{N}$ we denote

$$
R_{*}\left(\mathbf{p}_{0}\right)=\min \left\{\sup \left\{R>0: R \leq \frac{\pi}{2}\left[K_{B_{g}\left(\mathbf{p}_{0}, R\right)}\right]_{+}^{-\frac{1}{2}}\right\}, \frac{\ell\left(\mathbf{p}_{0}\right)}{4}\right\},
$$

where $\left[K_{B_{g}\left(\mathbf{p}_{0}, R\right)}\right]_{+}$is the supremum of sectional curvature over $B_{g}\left(\mathbf{p}_{0}, R\right)$ (compare with (9)) or +0 if the supremum is negative, $\ell\left(\mathbf{p}_{0}\right)$ is the infimum of lengths of maximal closed geodesics in $\mathscr{N}$ passing through $\mathbf{p}_{0}$, and $\pi$ is the length of a circle of radius $\frac{1}{2} \cdot R_{*}\left(\mathbf{p}_{0}\right)$ is positive and lower than both the convexity radius and half of the injectivity radius $\operatorname{inj}_{\mathscr{N}}\left(\mathbf{p}_{0}\right)$ [36, Section 6.3.2].

First, we prove

Lemma 4 Let $\mathbf{p}_{0} \in \mathscr{N}, \mathbf{u}_{0} \in W^{1, \infty}(\Omega)$. If $\mathbf{u}_{0}(\Omega) \subset \overline{B_{g}\left(\mathbf{p}_{0}, R\right)}$ with $\left.R \in\right] 0, R_{*}\left(\mathbf{p}_{0}\right)[$, then $\left.\mathbf{u}(t, \Omega) \subset \overline{B_{g}\left(\mathbf{p}_{0}, R\right)}, t \in\right] 0, T[$.

Proof We proceed by contradiction. Let $T_{*}=\inf \left\{t \in\left[0, T\left[: \mathbf{u}(t, \Omega) \not \subset \overline{B_{g}\left(\mathbf{p}_{0}, R\right)}\right\}\right.\right.$. Due to continuity of $\mathbf{u}$, there is a $\delta>0$ such that $\mathbf{u}(t, \Omega) \subset B_{g}\left(\mathbf{p}_{0}, R_{*}\left(\mathbf{p}_{0}\right)\right)$ for $t \in\left[0, T_{*}+\delta[\right.$. We choose on $B_{g}\left(\mathbf{p}_{0}, R_{*}\left(\mathbf{p}_{0}\right)\right)$ a polar coordinate system $\mathbf{p} \mapsto\left(p^{r}, p^{\vartheta^{1}}, \ldots, p^{\vartheta^{n-1}}\right)$ centered at $\mathbf{p}_{0}$. Due to the block diagonal form of the metric in these coordinates, (47) for the radial coordinate $p^{r}$ takes the form

$$
u_{t}^{r}=\operatorname{div} Z^{r}-\frac{1}{2} g_{\vartheta^{i} \vartheta^{j}, r}(\mathbf{u}) u_{x^{l}}^{\vartheta^{i}} Z_{l}^{\vartheta^{j}} .
$$

Here and in the following, $g_{\vartheta^{i} \vartheta^{j}, r}$ denotes the derivative of $g_{\vartheta^{i} \vartheta^{j}}$ (a function on $\mathscr{N}$ ) in direction $p^{r}$. Equation (49) is satisfied a.e., in the open $\operatorname{set}\{(t, \mathbf{x}) \in] 0, T_{*}+\delta[\times \Omega: \mathbf{u}(t, \mathbf{x}) \neq$ $\left.\mathbf{p}_{0}\right\}$. Furthermore, there holds (see the proof of Corollary 2.4 in [36, Chapter 6])

$$
\left(g_{\vartheta^{i} \vartheta^{j}, r}(\mathbf{p})\right)_{i, j=1}^{n-1} \geq \frac{2}{p^{r}} \cos \left(\left[K_{B_{g}\left(\mathbf{p}_{0}, R\right)}\right]_{+}^{\frac{1}{2}} p^{r}\right)\left(g_{\vartheta^{i} \vartheta^{j}}(\mathbf{p})\right)_{i, j=1}^{n-1} \quad \text { for } \quad \mathbf{p} \in \mathscr{N}
$$

as quadratic forms. Taking into account (5), (7), (49), (50) and recalling that $\mathbf{u}_{x^{l}}$ is a nonnegative multiple of $\mathbf{Z}_{l}$ for $l=1, \ldots, m$ we calculate

$$
\begin{aligned}
\frac{1}{2} \frac{\mathrm{d}}{\mathrm{d} t} \int_{\Omega}\left(u^{r}-R\right)_{+}^{2}= & \int_{\Omega}\left(u^{r}-R\right)_{+} u_{t}^{r} \leq-\int_{\left\{\mathbf{x} \in \Omega: u^{r}(\mathbf{x})>R\right\}}\left|\nabla u^{r}\right| \\
& -\int_{\Omega} \frac{\left(u^{r}-R\right)_{+}}{u^{r}}\left(\cos \frac{\pi}{2}\right) g_{\vartheta^{i} \vartheta^{j}}(\mathbf{u}) u_{x^{l}}^{\vartheta^{i}} Z_{l}^{\vartheta^{j}} \leq 0 .
\end{aligned}
$$

Next, we recall the notion of Riemannian center of mass. Let $R<R_{*}\left(\mathbf{p}_{0}\right), \mathbf{p}_{0} \in \mathscr{N}$. We say that $\mathbf{p}_{c} \in \overline{B_{g}\left(\mathbf{p}_{0}, R\right)}$ is a center of mass of a Radon measure $\mu$ on $\overline{B_{g}\left(\mathbf{p}_{0}, R\right)}$ if $\mathbf{p}_{c}$ is a minimizer of the function $f_{\mu}: \overline{B_{g}\left(\mathbf{p}_{0}, R\right)} \rightarrow[0, \infty[$ given by

$$
f_{\mu}(\mathbf{p})=\frac{1}{2} \int_{\mathscr{N}} \operatorname{dist}_{g}(\cdot, \mathbf{p})^{2} \mathrm{~d} \mu .
$$


A unique center of mass exists for any Radon measure on $\overline{B_{g}\left(\mathbf{p}_{0}, R\right)}$ and we have

$$
0=\mathrm{d} f_{\mu}\left(\mathbf{p}_{c}\right)=\int_{\frac{B_{g}\left(\mathbf{p}_{0}, R\right)}{}} \exp _{\mathbf{p}_{c}}^{-1} \mathrm{~d} \mu,
$$

where $\exp _{\mathbf{p}_{c}}^{-1}: B_{g}\left(\mathbf{p}_{c}, \operatorname{inj}_{\mathscr{N}}\left(\mathbf{p}_{c}\right)\right) \rightarrow T_{\mathbf{p}_{c}} \mathscr{N}$ denotes the logarithmic map at $\mathbf{p}_{c}$. In (52), we identified elements of $T_{\mathbf{p}_{c}}^{*} \mathscr{N}$ and $T_{\mathbf{p}_{c}} \mathscr{N}$ via $g$ [24, Section 1]. For $\mathbf{p}_{0} \in \mathscr{N}$, we denote

$$
\widetilde{R}_{*}\left(\mathbf{p}_{0}\right)=\frac{1}{2} \inf \left\{R_{*}(\mathbf{p}): \mathbf{p} \in B_{g}\left(\mathbf{p}_{0}, R_{*}\left(\mathbf{p}_{0}\right)\right)\right\} .
$$

We are ready to state

Lemma 5 Suppose that $\mathbf{u}_{0} \in W^{1, \infty}(\Omega)$ satisfies $\mathbf{u}_{0}(\Omega) \subset \overline{B_{g}\left(\mathbf{p}_{0}, R\right)}, \mathbf{p}_{0} \in \mathscr{N}, 0<R<$ $\widetilde{R}_{*}\left(\mathbf{p}_{0}\right)$. Let $\mathbf{p}_{c}(t)$ be the center of mass of the pushforward measure $\mu(t)=\mathbf{u}(t, \cdot)_{\#} \mathscr{L}^{m}$ on $\overline{B_{g}\left(\mathbf{p}_{0}, R\right)}$. There exists $C_{0}=C_{0}\left(\Omega, \mathscr{N}, \mathbf{p}_{0}\right)>0$ such that

$$
\frac{\mathrm{d}}{\mathrm{d} t} f_{\mu}\left(\mathbf{p}_{c}\right) \leq-C_{0} R^{\frac{2}{m}-1} f_{\mu}\left(\mathbf{p}_{c}\right)^{1-\frac{1}{m}}
$$

for $t>0$.

Proof We have

$$
f_{\mu(t)}\left(\mathbf{p}_{c}(t)\right)=\frac{1}{2} \int_{\Omega} \operatorname{dist}_{g}\left(\mathbf{u}(t, \cdot), \mathbf{p}_{c}(t)\right)^{2}=\frac{1}{2} \int_{\Omega} u^{r}(t, \cdot)^{2},
$$

where we have chosen polar coordinates centered at $\mathbf{p}_{c}(t)$. Employing (5), (7), (49), (50), (52) and observing that $\cos \left(\left[K_{B_{g}\left(\mathbf{p}_{c}, R\right)}\right]_{+}^{\frac{1}{2}} R\right) \geq \cos \left(\left[K_{B_{g}\left(\mathbf{p}_{c}, R_{*}\left(\mathbf{p}_{c}\right)\right)}\right]_{+}^{\frac{1}{2}} \frac{R_{*}\left(\mathbf{p}_{c}\right)}{2}\right) \in\left[\frac{\sqrt{2}}{2}, 1\right]$,

$$
\begin{aligned}
\frac{\mathrm{d}}{\mathrm{d} t} f_{\mu}\left(\mathbf{p}_{c}\right) & =\left\langle\mathrm{d} f_{\mu}\left(\mathbf{p}_{c}\right), \mathbf{p}_{c, t}\right\rangle_{T_{\mathbf{p}_{c}^{*} \mathscr{N}, T_{\mathbf{p}_{c}} \mathscr{N}}+\int_{\Omega} u^{r} u_{t}^{r}} \\
& \leq-\int_{\Omega}\left|\nabla u^{r}\right|-\cos \left(\left[K_{B_{g}\left(\mathbf{p}_{c}, R\right)}\right]_{+}^{\frac{1}{2}} R\right) \int_{\Omega} g_{\vartheta^{i} \vartheta^{j}}(\mathbf{u}) u_{x^{\vartheta^{i}}} Z_{l}^{\vartheta^{j}} \\
& \leq-\frac{\sqrt{2}}{2} \int_{\Omega}|\nabla \mathbf{u}|_{g} .
\end{aligned}
$$

This equation is rigorously justified by passing to the limit $R \rightarrow 0^{+}$in the weak formulation of (51) using Lebesgue monotone convergence theorem. Now, we choose on $B\left(\mathbf{p}_{c}, R_{*}\left(\mathbf{p}_{c}\right)\right)$ coordinate system $\mathbf{p} \mapsto \exp _{\mathbf{p}_{c}(t)}^{-1} \mathbf{p}=\left(p^{1}, \ldots, p^{n}\right)$. From (55) we obtain that there exists a constant $C_{1}=C_{1}\left(\mathscr{N}, \mathbf{p}_{0}\right)>0$ such that (recall that $\left.p^{r}=\sqrt{p^{i} p^{i}}\right)$

$$
\frac{\mathrm{d}}{\mathrm{d} t} \int_{\Omega} u^{i} u^{i} \leq-C_{1} \int_{\Omega} \sqrt{u_{x^{j}}^{i} u_{x^{j}}^{i}}
$$

Finally, applying Sobolev-Poincaré inequality (recall (52)):

$$
\left(\int_{\Omega} u^{i} u^{i}\right)^{1-\frac{1}{m}} \leq R^{1-\frac{2}{m}}\left(\int_{\Omega}\left(\sqrt{u^{i} u^{i}}\right)^{\frac{m}{m-1}}\right)^{1-\frac{1}{m}} \leq C_{2} R^{1-\frac{2}{m}} \int_{\Omega} \sqrt{u_{x^{j}}^{i} u_{x^{j}}^{i}}
$$

with $C_{2}=C_{2}(\Omega)>0$. Estimates (56), (57) add up to (54). 
Proof of Theorem 3 First of all, by Lemma 4, we obtain the bound $\mathbf{u}(t, \Omega) \subset \overline{B_{g}\left(\mathbf{p}_{0}, R\right)}$ if $\mathbf{u}_{0}(\Omega) \subset \overline{B_{g}\left(\mathbf{p}_{0}, R\right)}$ for $R<\widetilde{R}_{*}\left(\mathbf{p}_{0}\right)$ and any $t \in[0, T[$. Next, we deduce the estimate on extinction time from (54) by solving the ordinary differential inequality, which yields

$$
f_{\mu(t)}\left(\mathbf{p}_{c}(t)\right)^{\frac{1}{m}} \leq\left(f_{\mu(0)}\left(\mathbf{p}_{c}(0)\right)^{\frac{1}{m}}-\frac{1}{m} C_{0} R^{\frac{2}{m}-1} t\right)_{+},
$$

where

$$
f_{\mu(t)}\left(\mathbf{p}_{c}(t)\right)=\int_{\Omega} \operatorname{dist}\left(\mathbf{u}(t, \cdot), \mathbf{p}_{c}\right)^{2} .
$$

As $f_{\mu(0)}\left(\mathbf{p}_{c}(0)\right) \leq \frac{1}{2}|\Omega| R^{2}$, there is $\mathbf{u}_{*} \in \mathscr{N}$ such that $\mathbf{u}(t, \cdot) \equiv \mathbf{u}_{*}$ for $t \geq C R$, where $C=m\left(\frac{|\Omega|}{2}\right)^{\frac{1}{m}} C_{0}^{-1}$.

\section{Non-positive sectional curvature of the target}

This section is devoted to the proof of Theorem 4 .

Let $T>0$ and suppose that $\Omega$ is convex and $\mathscr{N}$ is a complete Riemannian manifold with $K_{\mathscr{N}} \leq 0$. In order to prove Theorem 4 without the assumption that there is a closed embedding of $\mathscr{N}$ into $\mathbb{R}^{N}$, we introduce a universal cover $\gamma: \widetilde{N} \rightarrow \mathscr{N}$ of $\mathscr{N}$ with a Riemannian manifold $(\widetilde{\mathscr{N}}, \widetilde{g})$. As a simply-connected Riemannian manifold of non-positive curvature, $\widetilde{\mathscr{N}}$ is diffeomorphic to $\mathbb{R}^{n}$ via the exponential map (this is the content of CartanHadamard theorem [10]). In other words, there is a global coordinate system on $\widetilde{\mathscr{N}}, \widetilde{\mathbf{p}} \mapsto$ $\exp _{\widetilde{\mathbf{p}}_{0}}^{-1} \widetilde{\mathbf{p}}=\left(\widetilde{p}^{1}, \ldots, \widetilde{p}^{n}\right)$. As $\Omega$ is topologically trivial, any function $\mathbf{u}_{0} \in C(\Omega, \mathscr{N})$ can be lifted preserving any Sobolev or Hölder regularity to $\widetilde{\mathbf{u}}_{0} \in C(\Omega, \widetilde{N})$ such that $\mathbf{u}_{0}=\gamma \circ \widetilde{\mathbf{u}}_{0}$. Then, assuming that $\Omega$ and $\mathbf{u}_{0}$ are of class $C^{3+\alpha}$ and $\mathbf{u}_{0}$ satisfies the compatibility conditions (37), (38) for $i=1, \ldots, n$, we consider the system

$$
\begin{aligned}
& \left.\widetilde{u}_{t}^{\varepsilon, i}=\operatorname{div} \frac{\nabla \widetilde{u}^{\varepsilon, i}}{\sqrt{\varepsilon^{2}+\left|\nabla \widetilde{\mathbf{u}}^{\varepsilon}\right|_{\tilde{g}}^{2}}}+\frac{1}{\sqrt{\varepsilon^{2}+\left|\nabla \widetilde{\mathbf{u}}^{\varepsilon}\right|_{\tilde{g}}^{2}}} \widetilde{\Gamma}_{j k}^{i}\left(\widetilde{\mathbf{u}}^{\varepsilon}\right) \widetilde{u}_{x^{l}}^{\varepsilon, j} \widetilde{u}_{x^{l}}^{\varepsilon, k} \text { in }\right] 0, T_{*}[\times \Omega, \\
& \left.\nabla \widetilde{u}^{\varepsilon, i} \cdot \boldsymbol{v}^{\Omega}=0 \text { in }\right] 0, T_{*}[\times \partial \Omega, \\
& \widetilde{u}^{\varepsilon, i}(0, \cdot)=\widetilde{u}_{0}^{i},
\end{aligned}
$$

$i=1, \ldots, n$. This system satisfies the assumptions of the Aquistapace-Terreni existence theorem (see Sect. 3.2), hence a unique solution exists for some $T_{*}>0$. Vector lengths $\left|\widetilde{\mathbf{u}}_{t}^{\varepsilon}\right| \widetilde{g}$ and $\left|\nabla \widetilde{\mathbf{u}}^{\varepsilon}\right| \tilde{g}$ are invariant under local isometries of the target manifold, and any Riemannian manifold is locally isometric to a submanifold in a Euclidean space. Therefore, we can repeat the proofs of Lemmata 1, 2 and 3 performing the computations in a neighbourhood of any point, obtaining bounds on $\left\|\widetilde{\mathbf{u}}_{t}^{\varepsilon}\right\|_{L^{2}(] 0, T_{*}[\times \Omega)}$ and $\left\|\nabla \widetilde{\mathbf{u}}^{\varepsilon}\right\|_{L^{\infty}(] 0, T_{*}[\times \Omega)}$ independent on $T_{*}$. Reasoning as in Sect. 3.2, the solution can be prolonged up to the arbitrary given $T$. Then, taking $\mathbf{u}^{\varepsilon}=\gamma \circ \widetilde{\mathbf{u}}^{\varepsilon}$, we obtain a solution to (20)-(22). Using the uniform bounds, we pass to the limit as in Sect. 4 obtaining a regular solution $\mathbf{u}$ to (2)-(4) with any $\mathbf{u}_{0} \in W^{1, \infty}(\Omega)$ in any convex $\Omega$.

Finally, we consider any lifting $\widetilde{\mathbf{u}}: \Omega \rightarrow \widetilde{\mathscr{N}}$ of $\mathbf{u}$ with $\widetilde{\mathbf{u}}_{t} \in L^{2}(] 0, T\left[\times \Omega, \mathbb{R}^{N}\right), \nabla \widetilde{\mathbf{u}} \in$ $L^{\infty}(] 0, T\left[\times \Omega, \mathbb{R}^{N}\right)$. As $R_{*}=+\infty$ for $\widetilde{N}$, arguments from Sect. 5 imply that $\widetilde{\mathbf{u}}$ becomes constant in finite time (if we take large enough $T$ ), and consequently the same holds for $\mathbf{u}=\gamma \circ \widetilde{\mathbf{u}}$. 


\section{The case where the domain is a Riemannian manifold}

Throughout this section, we assume that $(\mathscr{M}, \gamma)$ is an orientable, compact Riemannian manifold. Our aim is to prove Theorem 5.

Similarly as in Sect. 3, given $\varepsilon, T>0$ we first consider the following approximate system for $\mathbf{u}^{\varepsilon}:[0, T[\times \mathscr{M} \rightarrow \mathscr{N}:$

$$
\begin{aligned}
& \left.\mathbf{u}_{t}^{\varepsilon}=\pi_{\mathbf{u}^{\varepsilon}}\left(\operatorname{div}_{\gamma} \frac{\mathrm{du}^{\varepsilon}}{\sqrt{\varepsilon^{2}+\left|\mathrm{du}^{\varepsilon}\right|_{\gamma}^{2}}}\right) \text { in }\right] 0, T[\times \mathscr{M}, \\
& \mathbf{u}^{\varepsilon}(0, \cdot)=\mathbf{u}_{0} .
\end{aligned}
$$

Again, in what follows we drop the index $\varepsilon$ and denote

$$
\mathrm{Z}=\frac{\mathrm{d} \mathbf{u}}{\sqrt{\varepsilon^{2}+|\mathrm{d} \mathbf{u}|_{\gamma}^{2}}}, \quad v=\left(|\mathrm{d} \mathbf{u}|_{\gamma}^{2}+\varepsilon^{2}\right)^{\frac{1}{2}}, \quad v_{0}=\left(\left|\mathrm{d} \mathbf{u}_{0}\right|_{\gamma}^{2}+\varepsilon^{2}\right)^{\frac{1}{2}}
$$

Lemma 6 We have

$$
\sup _{t \in[0, T[} \int_{\mathscr{M}} v(t, \cdot)+\int_{0}^{T} \int_{\mathscr{M}} \mathbf{u}_{t}^{2} \leq \int_{\mathscr{M}} v_{0} .
$$

There exists $T_{\dagger}=T_{\dagger}\left(\right.$ Ric $\left.\left.\left._{\mathscr{M}}, K_{\mathscr{N}},\left\|v_{0}\right\|_{L^{\infty}}\right) \in\right] 0, \infty\right]$ and a non-decreasing function

$$
\left.M_{\text {Ric } \mathscr{M}, K_{\mathscr{N}},\left\|v_{0}\right\|_{L} \infty}:\right] 0, T_{\dagger}[\rightarrow] 0, \infty[
$$

such that for $t \in] 0, \min \left(T, T_{\dagger}\right)[$ there holds

$$
\|v(t, \cdot)\|_{L^{\infty}} \leq M_{R_{i c}, K_{\mathscr{N}},\left\|v_{0}\right\|_{L^{\infty}}}(t) .
$$

If $K_{\mathscr{N}} \leq 0, T_{\dagger}=+\infty$. If moreover Ric $\mathscr{M} \geq 0$, for $\left.t \in\right] 0, T\left[\right.$ there holds $\|v(t, \cdot)\|_{L^{\infty}} \leq$ $\left\|v_{0}\right\|_{L^{\infty}}$.

Proof We start by deriving a version of the Bochner formula (24) in our current setting. We calculate:

$$
\frac{1}{2} \frac{\mathrm{d}}{\mathrm{d} t}|\mathrm{~d} \mathbf{u}|_{\gamma}^{2}=\gamma^{a b} \mathbf{u}_{x^{a}} \cdot\left(\pi_{\mathbf{u}} \operatorname{div}_{\gamma} \mathbf{Z}\right)_{x^{b}}=\gamma^{a b} \mathbf{u}_{x^{a}} \cdot\left(\operatorname{div}_{\gamma} \mathbf{Z}\right)_{x^{b}}+\gamma^{a b} \mathbf{u}_{x^{a}} \cdot\left(\pi_{\mathbf{u}}\right)_{x^{b}} \operatorname{div}_{\gamma} \mathbf{Z} .
$$

Let us recall the expression of $\operatorname{div}_{\gamma}$ as the trace of covariant derivative [6, Lemma 2.6],

$$
\operatorname{div} \gamma \vartheta=\gamma^{a b} \vartheta_{a ; x^{b}}=\left(\gamma^{a b} \vartheta_{a}\right)_{; x^{b}}
$$

(note that covariant derivative of the metric vanishes) and the Ricci identity [39, Chapter 5]

$$
\vartheta_{a ; x^{b} x^{c}}-\vartheta_{a ; x^{c} x^{b}}=\vartheta_{d}\left(\mathscr{R}^{\mathscr{M}}\right)_{a b c}^{d}
$$

for the commutator of covariant derivatives of a 1-form $\vartheta$ on $\mathscr{M}$. Using (64), we obtain

$$
\left(\gamma^{c d} \mathbf{Z}_{c ; x^{d}}\right)_{x^{b}}=\gamma^{c d} \mathbf{Z}_{c ; x^{d} x^{b}}=\gamma^{c d} \mathbf{Z}_{c ; x^{b} x^{d}}+\gamma^{c d} \mathbf{Z}_{e}\left(\mathscr{R}^{\mathscr{M}}\right)_{c d b}^{e} .
$$

By antisymmetry of the Riemannian tensor and (16),

$$
\gamma^{c d}\left(\mathscr{R}^{\mathscr{M}}\right)_{c d b}^{e}=-\gamma^{e d}\left(\mathscr{R}^{\mathscr{M}}\right)_{d c b}^{c}=-\left(\mathscr{R} i c^{\mathscr{M}}\right)^{e f} \gamma_{f b} .
$$

An application of (63) yields

$$
\begin{aligned}
\gamma^{a b} \mathbf{u}_{x^{a}} \cdot \gamma^{c d} \mathbf{Z}_{c ; x^{b} x^{d}} & =\left(\gamma^{a b} \mathbf{u}_{x^{a}} \cdot \gamma^{c d} \mathbf{Z}_{c ; x^{b}}\right)_{; x^{d}}-\gamma^{a b} \mathbf{u}_{x^{a} ; x^{d}} \cdot \gamma^{c d} \mathbf{Z}_{c ; x^{b}} \\
& =\operatorname{div}_{\gamma}\left(\gamma^{a b} \mathbf{u}_{x^{a}} \cdot \mathbf{Z}_{; x^{b}}\right)-\gamma^{a b} \gamma^{c d} \mathbf{u}_{x^{d} ; x^{a}} \cdot \mathbf{Z}_{c ; x^{b}} .
\end{aligned}
$$


Combining (63), (65), (66) and (68) we obtain

$$
\gamma^{a b} \mathbf{u}_{x^{a}} \cdot\left(\operatorname{div}_{\gamma} \mathbf{Z}\right)_{x^{b}}=\operatorname{div}_{\gamma}\left(\gamma^{a b} \mathbf{u}_{x^{a}} \cdot \mathbf{Z}_{; x^{b}}\right)-\gamma^{a b} \gamma^{c d} \mathbf{u}_{x^{d} ; x^{a}} \cdot \mathbf{Z}_{c ; x^{b}}-\mathscr{R} i c^{\mathscr{M}}\left(\mathbf{Z}^{i}, \mathrm{~d} u^{i}\right) .
$$

From (62) and (68) we derive, proceeding as in the proof of Lemma 2, a Bochner-type formula involving only coordinate-invariant expressions:

$$
\begin{aligned}
\frac{1}{2} \frac{\mathrm{d}}{\mathrm{d} t}|\mathrm{~d} \mathbf{u}|_{\gamma}^{2}= & \operatorname{div}_{\gamma}\left(\gamma^{a b} \mathbf{u}_{x^{a}} \cdot \mathbf{Z}_{; x^{b}}\right)-\gamma^{a b} \gamma^{c d}\left(\pi_{\mathbf{u}} \mathbf{u}_{x^{d} ; x^{a}}\right) \cdot \mathbf{Z}_{c ; x^{b}} \\
& -\mathscr{R} i c^{\mathscr{M}}\left(\mathbf{Z}^{i}, \mathrm{~d} u^{i}\right)+\gamma^{a b} \gamma^{c d} \mathbf{Z}_{a} \cdot \mathscr{R}_{\mathbf{u}}^{\mathscr{N}}\left(\mathbf{u}_{x^{b}}, \mathbf{u}_{x^{c}}\right) \mathbf{u}_{x^{d}} .
\end{aligned}
$$

We take any $p>2$ and calculate $\frac{1}{p} \frac{\mathrm{d}}{\mathrm{d} t}|\mathrm{~d} \mathbf{u}|_{\gamma}^{p}$. Proceeding as in the proof of Lemma 3, appealing to (63) and the fact that covariant derivatives of the metric vanish (or just working in normal coordinates) we obtain

$$
\begin{aligned}
\frac{1}{p} \frac{\mathrm{d}}{\mathrm{d} t} v^{p} \leq & \operatorname{div}_{\gamma}\left(v^{p-2} \gamma^{a b} \mathbf{u}_{x^{a}} \cdot \mathrm{Z}_{; x^{b}}\right)-v^{p-3} \mathscr{R} i c^{\mathscr{M}}\left(\mathrm{d} u^{i}, \mathrm{~d} u^{i}\right) \\
& +v^{p-3} \gamma^{a b} \gamma^{c d} \mathbf{u}_{x^{a}} \cdot \mathscr{R}_{\mathbf{u}}^{\mathscr{N}}\left(\mathbf{u}_{x^{b}}, \mathbf{u}_{x^{c}}\right) \mathbf{u}_{x^{d}} \\
\leq & \operatorname{div}_{\gamma}\left(v^{p-2} \gamma^{a b} \mathbf{u}_{x^{a}} \cdot \mathrm{Z}_{; x^{b}}\right)-\operatorname{Ric}_{\mathscr{M}} v^{p-1}+K_{\mathscr{N}} v^{p+1}
\end{aligned}
$$

Next, we integrate (70) over $\mathscr{M}$. As $\mathscr{M}$ is compact and orientable, the term

$$
\int_{\mathscr{M}} \operatorname{div}_{\gamma}\left(v^{p-2} \gamma^{a b} \mathbf{u}_{x^{a}} \cdot \mathbf{Z}_{; x^{b}}\right) \mathrm{d} \mu_{\gamma}
$$

vanishes due to Stokes theorem. We are led to the following estimate:

$$
\begin{aligned}
\frac{1}{p} \frac{\mathrm{d}}{\mathrm{d} t} \int_{\mathscr{M}} v^{p} \mathrm{~d} \mu_{\gamma} & \leq-\operatorname{Ric}_{\mathscr{M}} \int_{\mathscr{M}} v^{p-1} \mathrm{~d} \mu_{\gamma}+K_{\mathscr{N}} \int_{\mathscr{M}} v^{p+1} \mathrm{~d} \mu_{\gamma} \\
& \leq-\operatorname{Ric}_{\mathscr{M}}^{-} \mu_{\gamma}(\mathscr{M})^{\frac{1}{p}}\left(\int_{\mathscr{M}} v^{p} \mathrm{~d} \mu_{\gamma}\right)^{1-\frac{1}{p}}+K_{\mathscr{N}}^{+}\|v\|_{L^{\infty}} \int_{\mathscr{M}} v^{p} \mathrm{~d} \mu_{\gamma},
\end{aligned}
$$

where we have used Hölder inequality and denoted $\operatorname{Ric}_{\mathscr{M}}^{-}=\min \left(\right.$ Ric $\left._{\mathscr{M}}, 0\right), K_{\mathscr{N}}^{+}=$ $\max \left(K_{\mathscr{N}}, 0\right)$. Thus,

$$
\frac{\mathrm{d}}{\mathrm{d} t}\left(\int_{\mathscr{M}} v^{p} \mathrm{~d} \mu_{\gamma}\right)^{\frac{1}{p}} \leq-\operatorname{Ric}_{\mathscr{M}}^{-} \mu_{\gamma}(\mathscr{M})^{\frac{1}{p}}+K_{\mathscr{N}}^{+}\|v\|_{L^{\infty}}\left(\int_{\mathscr{M}} v^{p} \mathrm{~d} \mu_{\gamma}\right)^{\frac{1}{p}} .
$$

Passing to the limit $p \rightarrow \infty$,

$$
\frac{\mathrm{d}}{\mathrm{d} t}\|v\|_{L^{\infty}} \leq-\operatorname{Ric}_{\mathscr{M}}^{-}+K_{\mathscr{N}}^{+}\|v\|_{L^{\infty}}^{2} .
$$

We let $M_{\text {Ric } \mathscr{M}}, K_{\mathscr{N}},\left\|v_{0}\right\|_{L^{\infty}}$ be the locally existing solution to

$$
\frac{\mathrm{d} M}{\mathrm{~d} t}=-\operatorname{Ric}_{\mathscr{M}}^{-}+K_{\mathscr{N}}^{+} M^{2}
$$

with initial datum $\left\|v_{0}\right\|_{L^{\infty}}$, and let $T_{\dagger}$ be the maximal time of existence of $M_{R i c_{\mathscr{M}}, K_{\mathscr{N}},\left\|v_{0}\right\|_{L} \infty}$, completing the proof.

Proposition 2 Let $\mathbf{u}_{0} \in C^{3+\alpha}(\mathscr{M}, \mathscr{N})$. There exist $T_{\dagger}=T_{\dagger}\left(\operatorname{Ric}_{\mathscr{M}}, K_{\mathscr{N}},\left\|\nabla \mathbf{u}_{0}\right\|_{L^{\infty}}\right)>0$ and unique solution $\mathbf{u} \in C_{l o c}^{\frac{3+\alpha}{2}, 3+\alpha}\left(\left[0, T_{\dagger}[\times \mathscr{M}, \mathscr{N})\right.\right.$ to the system $(58),(59)$. 
Proof Let $\mathbf{u}_{0} \in C^{3+\alpha}(\mathscr{M}, \mathscr{N})$. As in [23, Section 3], we show that there exists $T>0$ and unique solution $\mathbf{u} \in C^{1}\left([0, T], C^{\alpha}(\mathscr{M}, \mathscr{N})\right) \cap C\left([0, T], C^{2+\alpha}(\mathscr{M}, \mathscr{N})\right)$ to $(58),(59)$. Using linear theory [26], we rise regularity of the solution to $C^{\frac{3+\alpha}{2}, 3+\alpha}([0, T[\times \mathscr{M}, \mathscr{N})$. Then, using the uniform bound on du in $L^{\infty}$ from Lemma 6 we show that the solution can be extended to $\left[0, T_{\dagger}[\right.$ as in the proof of Proposition 1.

Proof of Theorem 5 The proof of uniqueness follows along the lines of the proof of Theorem 1. An important point is that integration by parts is allowed because $\mathscr{M}$ is orientable.

Given any initial datum $\mathbf{u}_{0} \in W^{1, \infty}(\mathscr{M}, \mathscr{N})$, we take an approximating family $\left(\mathbf{u}_{0}^{\varepsilon}\right) \subset C^{3+\alpha}(\mathscr{M}, \mathscr{N})$ such that $\mathbf{u}_{0}^{\varepsilon} \rightarrow \mathbf{u}_{0}$ as $\varepsilon \rightarrow 0^{+}$in $C(\mathscr{M}, \mathscr{N})$ and $\left\|\mathrm{d} \mathbf{u}_{0}^{\varepsilon}\right\|_{L^{\infty}} \rightarrow$ $\left\|\mathrm{d} \mathbf{u}_{0}\right\|_{L^{\infty}}$. Proposition 2 generates a family $\left(\mathbf{u}^{\varepsilon}\right)$, where $\mathbf{u}^{\varepsilon}$ solves (58) with initial datum $\mathbf{u}_{0}^{\varepsilon}$. This family satisfies uniform bounds on $\left(\mathbf{u}_{t}^{\varepsilon}\right)$ in $L^{2}(] 0, T_{\dagger}\left[\times \mathscr{M}, \mathbb{R}^{N}\right)$ and on $\left(\mathrm{d} \mathbf{u}^{\varepsilon}\right)$ in $L_{\text {loc }}^{\infty}\left(\left[0, T_{\dagger}\left[\times T^{*} \mathscr{M} \times \mathbb{R}^{N}\right)\right.\right.$. Using these bounds, we pass to the limit as in Sect. 4 and obtain the regular solution $(\mathbf{u}, Z)$ to (12) in $\left[0, T_{\dagger}\left[\right.\right.$. Recall that if $K_{\mathscr{N}} \leq 0, T_{\dagger}=+\infty$.

Now we assume that $K_{\mathscr{N}} \leq 0$ and Ric $_{\mathscr{M}} \geq 0$. In this case we have

$$
\mathbf{u}_{t} \in L^{2}(] 0, \infty\left[\times \mathscr{M}, \mathbb{R}^{N}\right), \quad\|\mathrm{d} \mathbf{u}(t, \cdot)\|_{L^{\infty}} \leq\left\|\mathrm{d} \mathbf{u}_{0}\right\|_{L^{\infty}} \quad \text { in a. e. } t>0 .
$$

Therefore, we can choose a sequence of time instances $\left.\left(t_{k}\right) \subset\right] 0, \infty\left[, t_{k} \rightarrow \infty\right.$ such that there exists $\mathbf{u}_{*} \in W^{1, \infty}(\mathscr{M}, \mathscr{N})$ with

$$
\mathbf{u}\left(t_{k}, \cdot\right) \rightarrow \mathbf{u}_{*} \text { in } C(\mathscr{M}, \mathscr{N}), \quad \mathbf{u}_{t}\left(t_{k}, \cdot\right) \rightarrow \mathbf{0} \quad \text { in } L^{2}\left(\mathscr{M}, \mathbb{R}^{N}\right),
$$

and

$$
\mathbf{u}_{t}\left(t_{k}, \cdot\right)=\pi_{\mathbf{u}\left(t_{k}, \cdot\right)}\left(\operatorname{div}_{\gamma} \mathrm{Z}\left(t_{k}, \cdot\right)\right), \quad \mathrm{Z}\left(t_{k}, \cdot\right) \in \frac{\mathrm{du}}{|\mathrm{d} \mathbf{u}|_{\gamma}}\left(t_{k}, \cdot\right) \quad \mu_{\gamma}-\text { a. e. in } \mathscr{M} .
$$

The first item in (74) can be rewritten as

$$
\mathbf{u}_{t}\left(t_{k}, \cdot\right)=\operatorname{div}_{\gamma} \mathbf{Z}\left(t_{k}, \cdot\right)+\gamma^{a b} \mathscr{A}_{\mathbf{u}\left(t_{k}, \cdot\right)}\left(\mathbf{u}_{x^{a}}\left(t_{k}, \cdot\right), \mathbf{Z}_{b}\left(t_{k}, \cdot\right)\right),
$$

hence (72) implies that the sequence $\operatorname{div}_{\gamma} \mathrm{Z}\left(t_{k}, \cdot\right)$ is uniformly bounded in $L^{2}\left(\mathscr{M}, \mathbb{R}^{N}\right)$. The second item in (74) is equivalent to

$$
\begin{aligned}
& \pi_{\mathbf{u}\left(t_{k}, \cdot\right)}^{\perp} \mathrm{Z}\left(t_{k}, \cdot\right)=0, \quad\left|\mathrm{Z}\left(t_{k}, \cdot\right)\right|_{\gamma} \leq 1, \\
& \quad \gamma^{a b} \mathbf{u}_{x^{a}}\left(t_{k}, \cdot\right) \cdot \mathbf{Z}_{b}\left(t_{k}, \cdot\right)=\left|\operatorname{du}\left(t_{k}, \cdot\right)\right|_{\gamma} \quad \mu_{\gamma}-\text { a. e. in } \mathscr{M} .
\end{aligned}
$$

Hence, there exists $Z_{*} \in L^{\infty}\left(T^{*} \mathscr{M} \times \mathbb{R}^{N}\right)$ satisfying $\operatorname{div}_{\gamma} Z_{*} \in L^{\infty}\left(\mathscr{M}, \mathbb{R}^{\mathscr{N}}\right)$ and (possibly decimating the sequence $\left.\left(t_{k}\right)\right)$

$$
\begin{aligned}
& \mathrm{Z}\left(t_{k}, \cdot\right) \stackrel{*}{\rightarrow} \mathrm{Z}_{*} \text { in } L^{\infty}\left(T^{*} \mathscr{M} \times \mathbb{R}^{N}\right), \quad \operatorname{div}_{\gamma} \mathrm{Z}\left(t_{k}, \cdot\right) \rightarrow \operatorname{div}_{\gamma} \mathrm{Z}_{*} \text { in } L^{2}\left(\mathscr{M}, \mathbb{R}^{N}\right), \\
& \pi_{\mathbf{u}_{*}}^{\perp} \mathrm{Z}_{*}=0, \quad\left|\mathrm{Z}_{*}\right|_{\gamma} \leq 1 \quad \mu_{\gamma}-\text { a. e. in } \mathscr{M} .
\end{aligned}
$$

Using a standard div-curl reasoning and weak-star convergence of $\mathbf{u}\left(t_{k}, \cdot\right)$ in $W^{1, \infty}(\mathscr{M}, \mathscr{N})$ we also obtain

$$
\left|\mathrm{d} \mathbf{u}_{*}\right|_{\gamma} \leq \liminf \left|\operatorname{du}\left(t_{k}, \cdot\right)\right|_{\gamma}=\gamma^{a b} \mathbf{u}_{*, x^{a}} \cdot \mathbf{Z}_{*, b} \leq\left|\mathrm{d} \mathbf{u}_{*}\right|_{\gamma} \quad \mu_{\gamma}-\text { a. e. in } \mathscr{M} .
$$

This together with (76) yields the second item of (17). The first item of (17) is produced by passing to the limit in the first item of (74) using (73), (75).

Acknowledgements The authors are grateful to the anonymous reviewer for his or her helpful comments. M. $Ł$. has been supported by the Grant No. 2014/13/N/ST1/02622 of the National Science Centre, Poland. S. M. has been partially supported by the Spanish MINECO and FEDER Project MTM2015-70227-P as well as the Simons Foundation Grant 346300 and the Polish Government MNiSW 2015-2019 matching fund. 
Open Access This article is distributed under the terms of the Creative Commons Attribution 4.0 International License (http://creativecommons.org/licenses/by/4.0/), which permits unrestricted use, distribution, and reproduction in any medium, provided you give appropriate credit to the original author(s) and the source, provide a link to the Creative Commons license, and indicate if changes were made.

\section{Appendix: Technical lemmata}

Lemma A.1 Let $(\mathscr{N}, g)$ be a closed embedded Riemannian submanifold in the Euclidean space $\mathbb{R}^{N}$. There exists a Riemannian metric $h$ on $\mathbb{R}^{N}$ such that $(\mathscr{N}, g)$ is a totally geodesic Riemannian submanifold of $\left(\mathbb{R}^{N}, h\right)$.

Proof Let $R>0$. As $\mathscr{N}$ is a closed submanifold of $\mathbb{R}^{N}, \mathscr{N} \cap \overline{B(0, R)}$ is compact. Hence, there is a non-increasing function $\left.R \mapsto \delta_{R} \in\right] 0,1[$ such that

$$
N_{R, \delta}=\left\{\mathbf{y}+\mathbf{n}: \mathbf{y} \in \mathscr{N} \cap B(0, R), \mathbf{n} \in T_{\mathbf{y}} \mathscr{N}^{\perp},|\mathbf{n}|<\delta\right\}
$$

is a tubular neighborhood of $\mathscr{N} \cap B(0, R)$ in $\mathbb{R}^{N}$ that does not intersect $\mathscr{N} \backslash B(0, R)$ for $\delta \in] 0, \delta_{R}$ [. Identifying $T_{\mathbf{y}+\mathbf{n}} N_{R, \delta_{R}}$ with $T_{\mathbf{y}} \mathscr{N} \times \mathbb{R}^{N-n}$, we define a Riemannian metric $h^{R}$ on $N_{R, \delta_{R}}$ as follows:

$$
h_{\mathbf{p}+\mathbf{n}}^{R}\left(\mathbf{w}_{1}+\mathbf{w}_{1}^{\prime}, \mathbf{w}_{2}+\mathbf{w}_{2}^{\prime}\right)=g_{\mathbf{p}}\left(\mathbf{w}_{1}, \mathbf{w}_{2}\right)+\mathbf{w}_{1}^{\prime} \cdot \mathbf{w}_{2}^{\prime}
$$

for $\mathbf{p} \in \mathscr{N} \cap B(0, R),|\mathbf{n}|<\delta_{R}, \mathbf{w}_{1}, \mathbf{w}_{2} \in T_{\mathbf{p}} \mathscr{N}, \mathbf{w}_{1}^{\prime}, \mathbf{w}_{2}^{\prime} \in \mathbb{R}^{N-n}$. Next, we define the tubular neighborhood of $\mathscr{N}$

$$
\mathscr{T}=\bigcup_{k=1}^{\infty} N_{k, \frac{1}{2}} \delta_{k+1}
$$

so that

$$
\left\{\mathbb{R}^{N} \backslash \overline{\mathscr{T}}, N_{1, \delta_{1}}, N_{2, \delta_{2}}, \ldots\right\}
$$

is an open cover of $\mathbb{R}^{N}$. Indeed, if $\mathbf{z} \notin \mathbb{R}^{N} \backslash \overline{\mathscr{T}}$, i.e., $\mathbf{z} \in \overline{\mathscr{T}}$, then letting $k_{0}$ be the smallest integer bound of $|\mathbf{z}|$, we have

$$
\mathbf{z} \in \overline{\mathscr{T}} \cap B\left(0, k_{0}+1\right) \subset \bigcup_{k=1}^{\overline{k_{0}+1} N_{k, \frac{1}{2} \delta_{k+1}}}=\bigcup_{k=1}^{k_{0}+1} \overline{N_{k, \frac{1}{2}} \delta_{k+1}} .
$$

Here, we used the fact that $U \cap \overline{\bigcup_{k=1}^{\infty} A_{k}} \subset \overline{\bigcup_{k=1}^{\infty} U \cap A_{k}}$ for any sequence of sets $A_{k}$ and open set $U$. Hence, by definition of $k_{0}, \mathbf{z} \in \overline{N_{k_{0}, \frac{1}{2} \delta_{k_{0}+1}}}$. Therefore

$$
\mathbf{z}=\mathbf{y}+\mathbf{n} \text { with }|\mathbf{n}| \leq \frac{1}{2} \delta_{k_{0}+1}<\delta_{k_{0}+1} \text { and } \mathbf{y} \in \overline{B\left(0, k_{0}\right)} \subset B\left(0, k_{0}+1\right),
$$

that is, $\mathbf{z} \in N_{k_{0}+1, \delta_{k_{0}+1}}$.

We take a smooth partition of unity $\left\{\varphi_{0}, \varphi_{1}, \varphi_{2}, \ldots\right\}$ subordinate to this cover (a construction of a partition of unity subordinate to an infinite open cover can be found in [43, Appendix C]) and define

$$
h_{\mathbf{y}}\left(\mathbf{v}_{1}, \mathbf{v}_{2}\right)=\varphi_{0}(\mathbf{y}) \mathbf{v}_{1} \cdot \mathbf{v}_{2}+\sum_{k=1}^{\infty} \varphi_{k}(\mathbf{y}) h_{\mathbf{y}}^{k}\left(\mathbf{v}_{1}, \mathbf{v}_{2}\right)
$$

for $\mathbf{y} \in \mathbb{R}^{N}$. It is easy to check that $(\mathscr{N}, g)$ is a totally geodesic submanifold in $\left(\mathbb{R}^{N}, h\right)$. 
Lemma A.2 Let $\mathbf{u}_{0} \in W^{1, \infty}(\Omega, \mathscr{N})$. There exists a family $\left.\left(\mathbf{u}_{0, \varepsilon}\right) \subset C^{\infty}(\bar{\Omega}, \mathscr{N}), \varepsilon \in\right] 0, \varepsilon_{0}[$, $\varepsilon_{0}>0$ such that

$-\mathbf{u}_{0, \varepsilon} \rightarrow \mathbf{u}_{0}$ in $C\left(\bar{\Omega}, \mathbb{R}^{N}\right)$ as $\varepsilon \rightarrow 0^{+}$,

- $\left\|\nabla \mathbf{u}_{0, \varepsilon}\right\|_{L^{\infty}} \rightarrow\left\|\nabla \mathbf{u}_{0}\right\|_{L^{\infty}}$ as $\varepsilon \rightarrow 0^{+}$,

- $\mathbf{u}_{0, \varepsilon}$ satisfy compatibility conditions (37), (38) for $\left.\varepsilon \in\right] 0, \varepsilon_{0}[$.

Proof As $\partial \Omega$ is a compact smooth submanifold of $\mathbb{R}^{m}$, there is $\varepsilon_{0}^{\prime}>0$ and a tubular neighbourhood of $\partial \Omega$

$$
T=\left\{\mathbf{y}+r \boldsymbol{v}^{\Omega}, \mathbf{y} \in \partial \Omega, r \in\right]-\varepsilon_{0}^{\prime}, \varepsilon_{0}^{\prime}[\} .
$$

We extend $\mathbf{u}_{0}$ to $\mathbf{w} \in W^{1, \infty}(\Omega \cup T, \mathscr{N})$ putting

$$
\mathbf{w}\left(\mathbf{y}+r v^{\Omega}(\mathbf{y})\right)=\mathbf{y}
$$

for $r \in\left[0, \varepsilon_{0}^{\prime}[\right.$. For any $\varepsilon \in] 0, \varepsilon_{0}^{\prime}[$ we define

$$
\Omega_{\varepsilon}=\{\mathbf{x} \in \Omega: \operatorname{dist}(\mathbf{x}, \partial \Omega)>\varepsilon\} .
$$

Mollifying $\mathbf{w}$ as in $[24$, Theorems $4.4,4.6]$ we produce a family of maps $\left(\mathbf{w}_{\varepsilon}\right)_{\varepsilon \in] 0, \varepsilon_{0}[}, \varepsilon_{0} \in$ ] $0, \varepsilon_{0}^{\prime}\left[, \mathbf{w}_{\varepsilon} \in C^{\infty}(\bar{\Omega}, \mathscr{N})\right.$ such that $\mathbf{w}_{\varepsilon} \rightarrow \mathbf{u}_{0}$ in $C(\bar{\Omega}, \mathscr{N})$ and $\left\|\nabla \mathbf{w}_{\varepsilon}\right\|_{L^{\infty}} \rightarrow\left\|\nabla \mathbf{u}_{0}\right\|_{L^{\infty}}$ as $\varepsilon \rightarrow 0^{+}$.

Now, let $\eta_{\varepsilon} \in C^{\infty}(] 0, \varepsilon[] 0,, \varepsilon[)$ satisfy the conditions

$-\eta_{\varepsilon}(r)=r$ for $r \in\left[\frac{\varepsilon}{2}, \varepsilon[\right.$,

$-\eta_{\varepsilon}^{\prime}(r)=0$ for $\left.\left.r \in\right] 0, \frac{\varepsilon}{4}\right]$,

$-0 \leq \eta^{\prime} \leq 1$

We define $\Phi_{\varepsilon} \in C^{\infty}(\Omega, \Omega)$ by

$$
\Phi_{\varepsilon}(\mathbf{x})= \begin{cases}\mathbf{y}-\eta_{\varepsilon}(r) \boldsymbol{v}^{\Omega} & \text { if } \quad \mathbf{x}=\mathbf{y}-r \boldsymbol{v}^{\Omega} \in \Omega \backslash \Omega_{\varepsilon}, \\ \mathbf{x} & \text { if } \quad \mathbf{x} \in \Omega_{\varepsilon} .\end{cases}
$$

It is easy to see that $\mathbf{u}_{0, \varepsilon}=\mathbf{w}_{\varepsilon} \circ \Phi_{\varepsilon}$ satisfies the desired conditions.

Lemma A.3 Let $\Omega \subset \mathbb{R}^{m}$ be open and convex. There exists a family $\left(\Omega_{\varepsilon}\right)$ of open, convex sets with smooth boundary such that $\Omega_{\varepsilon} \subset \Omega$ for $\left.\varepsilon \in\right] 0, \varepsilon_{0}\left[, \varepsilon_{0}>0\right.$ and the Hausdorff distance of $\Omega_{\varepsilon}$ from $\Omega$ tends to zero as $\varepsilon \rightarrow 0^{+}$.

Proof Let $d$ denote the signed distance function of $\Omega$, i.e.,

$$
d(\mathbf{x})=\operatorname{dist}(\mathbf{x}, \Omega)-\operatorname{dist}\left(\mathbf{x}, \mathbb{R}^{m} \backslash \Omega\right) \text { for } \quad \mathbf{x} \in \mathbb{R}^{m} .
$$

This function is convex and satisfies

$$
|d(\mathbf{x})-d(\mathbf{y})| \leq|\mathbf{x}-\mathbf{y}| \text { for } \mathbf{x}, \mathbf{y} \text { in } \mathbb{R}^{N} .
$$

Let $\left(\varphi_{\varepsilon}\right)_{\varepsilon>0}$ be a standard family of mollifying kernels such that

$$
\operatorname{supp} \varphi_{\varepsilon} \subset B(\mathbf{0}, \varepsilon)
$$

and denote $d_{\varepsilon}=\varphi_{\varepsilon} * d$. It is easy to check that $d_{\varepsilon}$ is smooth and convex. Let us further denote

$$
\Omega_{\varepsilon}=\left\{\mathbf{x} \in \mathbb{R}^{m}: d_{\varepsilon}(\mathbf{x})<-\varepsilon\right\}
$$


As a sublevel set of a convex function, $\Omega_{\varepsilon}$ is convex. Now, denote by $r_{\Omega}$ the inradius of $\Omega$, equivalently $r_{\Omega}=|\min d|$. Take $\varepsilon_{0}=\frac{r_{\Omega}}{3}$ and assume $\varepsilon<\varepsilon_{0}$. Suppose that $d(\mathbf{x}) \geq 0$. Due to (77), (78), we have

$$
d_{\varepsilon}(\mathbf{x})=\int_{B(\mathbf{x}, \varepsilon)} \varphi_{\varepsilon}(\mathbf{x}-\mathbf{y}) d(\mathbf{y}) \mathrm{d} \mathbf{y}>-\varepsilon .
$$

Hence, $\bar{\Omega}_{\varepsilon} \subset \Omega$. Similarly, if $d(\mathbf{x}) \leq-2 \varepsilon$, then $d_{\varepsilon}(\mathbf{x})<-\varepsilon$. This in turn implies that

$$
\operatorname{dist}\left(\partial \Omega_{\varepsilon}, \partial \Omega\right)=\min \left\{-d(\mathbf{x}): d_{\varepsilon}(\mathbf{x})=-\varepsilon\right\}<2 \varepsilon .
$$

Denoting by $\mathbf{x}_{\Omega}$ the center of any circle inscribed in $\bar{\Omega}$,

$$
\min d_{\varepsilon} \leq \int_{B\left(\mathbf{x}_{\Omega}, \varepsilon\right)} \varphi_{\varepsilon}\left(\mathbf{x}_{\Omega}-\mathbf{y}\right) d(\mathbf{y}) \mathrm{d} \mathbf{y}<-r_{\Omega}+\varepsilon<-2 \varepsilon .
$$

Recall that a critical point of a smooth convex function on $\mathbb{R}^{m}$ is necessarily its global (possibly improper) minimum. Hence, by virtue of (79), (80), $\Omega_{\varepsilon}$ does not contain critical points of $d_{\varepsilon}$, and so it is a smooth hypersurface. Finally, (79) implies the Hausdorff convergence of $\Omega_{\varepsilon}$ to $\Omega$ as $\varepsilon \rightarrow 0^{+}$.

\section{References}

1. Acquistapace, P., Terreni, B.: On quasilinear parabolic systems. Math. Ann. 282(2), 315-335 (1988)

2. Agmon, S., Douglis, A., Nirenberg, L.: Estimates near the boundary for solutions of elliptic partial differential equations satisfying general boundary conditions II. Commun. Pure Appl. Math. 17, 35-92 (1964)

3. Andreu-Vaillo, F., Caselles, V., Mazón, J.M.: Parabolic quasilinear equations minimizing linear growth functionals. In: Progress in Mathematics, vol. 223. Birkhäuser, Basel (2004)

4. Anzellotti, G.: Pairings between measures and bounded functions and compensated compactness. Ann. Mat. Pura Appl. (4) 135, 293-318 (1983)

5. Bonforte, M., Figalli, A.: Total variation flow and sign fast diffusion in one dimension. J. Differ. Equ. 252(8), 4455-4480 (2012)

6. Calin, O., Chang, D.-C.: Geometric mechanics on riemannian manifolds: applications to partial differential equations. Birkhäuser Boston Inc, Boston (2005)

7. Caselles, Vicent, Chambolle, Antonin, Novaga, Matteo: Regularity for solutions of the total variation denoising problem. Rev. Mat. Iberoam. 27(1), 233-252 (2011)

8. Dal Passo, Roberta, Giacomelli, Lorenzo, Moll, Salvador: Rotationally symmetric 1-harmonic maps from $D^{2}$ to $S^{2}$. Calc. Var. Partial Differ. Equ. 32(4), 533-554 (2008)

9. Di Castro, Agnese, Giacomelli, Lorenzo: The 1-harmonic flow with values into a smooth planar curve. Nonlinear Anal. 143, 174-192 (2016)

10. do Carmo, M.P.: Riemannian geometry. In: Mathematics: Theory and Applications. Birkhäuser Boston Inc., Boston (1992). Translated from the second Portuguese edition by Francis Flaherty

11. Eells Jr., J., Sampson, J.H.: Harmonic mappings of Riemannian manifolds. Am. J. Math. 86, 109-160 (1964)

12. Fardoun, A., Regbaoui, R.: Heat flow for $p$-harmonic maps between compact Riemannian manifolds. Indiana Univ. Math. J. 51(6), 1305-1320 (2002)

13. Fardoun, A., Regbaoui, R.: Heat flow for $p$-harmonic maps with small initial data. Calc. Var. Partial Differ. Equ. 16(1), 1-16 (2003)

14. Giacomelli, L., Mazón, J.M., Moll, S.: The 1-harmonic flow with values into $\mathbb{S}^{1}$. SIAM J. Math. Anal. 45(3), 1723-1740 (2013)

15. Giacomelli, L., Mazón, J.M., Moll, S.: The 1-harmonic flow with values in a hyperoctant of the $N$-sphere. Anal. PDE 7(3), 627-671 (2014)

16. Giacomelli, L., Moll, S.: Rotationally symmetric 1-harmonic flows from $D^{2}$ to $S^{2}$ : local well-posedness and finite time blowup. SIAM J. Math. Anal. 42(6), 2791-2817 (2010)

17. Giga, Y., Kashima, Y., Yamazaki, N.: Local solvability of a constrained gradient system of total variation. Abstr. Appl. Anal. 8, 651-682 (2004) 
18. Giga, Y., Kohn, R.V.: Scale-invariant extinction time estimates for some singular diffusion equations. Discrete Contin. Dyn. Syst. 30(2), 509-535 (2011)

19. Giga, Y., Kuroda, H.: A counterexample to finite time stopping property for one-harmonic map flow. Commun. Pure Appl. Anal. 14(1), 121-125 (2015)

20. Giga, Y., Kuroda, H., Yamazaki, N.: Global solvability of constrained singular diffusion equation associated with essential variation. In: Free Boundary Problems, Volume 154 of International Series of Numerical Mathematics, pp. 209-218. Birkhäuser, Basel (2007)

21. Greene, R.E.: Isometric embeddings of Riemannian and pseudo-Riemannian manifolds. In: Memoirs of the American Mathematical Society, No. 97. American Mathematical Society, Providence, RI (1970)

22. Haškovec, J., Schmeiser, C.: A note on the anisotropic generalizations of the Sobolev and Morrey embedding theorems. Monatsh. Math. 158(1), 71-79 (2009)

23. Hungerbühler, N.: $m$-harmonic flow. Ann. Scuola Norm. Sup. Pisa Cl. Sci. (4) 24(4), 593-631 (1997)

24. Karcher, H.: Riemannian center of mass and mollifier smoothing. Commun. Pure Appl. Math. 30(5), 509-541 (1977)

25. Kielak, K., Mucha, P.B., Rybka, P.: Almost classical solutions to the total variation flow. J. Evol. Equ. 13(1), 21-49 (2013)

26. Ladyzhenskaya, O.A., Solonnikov, V.A., Ural'tseva. N.N.: Lineynye i kvazilineynye uravneniya parabolicheskogo tipa. Izdatel'stvo "Nauka" (1967)

27. Łasica, M., Moll, S., Mucha, P.B.: Total variation denoising in $l^{1}$ anisotropy. SIAM J. Imaging Sci. 10(4), 1691-1723 (2017)

28. Lellmann, J., Strekalovskiy, E., Koetter, S., Cremers, D.: Total variation regularization for functions with values in a manifold. In: The IEEE International Conference on Computer Vision (ICCV) (December 2013)

29. Lin, F., Wang, C.: The Analysis of Harmonic Maps and Their Heat Flows. World Scientific Publishing Co. Pte. Ltd., Hackensack (2008)

30. Lunardi, A.: Analytic semigroups and optimal regularity in parabolic problems. In: Progress in Nonlinear Differential Equations and their Applications, vol. 16. Birkhäuser, Basel (1995)

31. Mazowiecka, Katarzyna, Schikorra, Armin: Fractional div-curl quantities and applications to nonlocal geometric equations. J. Funct. Anal. 275(1), 1-44 (2018)

32. Misawa, M.: Existence and regularity results for the gradient flow for $p$-harmonic maps. Electron. J. Differ. Equ. 36, 1-17 (1998)

33. Misawa, M.: Existence of a classical solution for linear parabolic systems of nondivergence form. Comment. Math. Univ. Carolin. 45(3), 475-482 (2004)

34. Nash, John: The imbedding problem for Riemannian manifolds. Ann. Math. 2(63), $20-63$ (1956)

35. Palais, R.S.: Foundations of Global Non-linear Analysis. W. A. Benjamin, Inc., New York (1968)

36. Petersen, P.: Riemannian geometry. In: Graduate Texts in Mathematics, vol. 171. Springer, New York (1998)

37. Schlag, W.: Schauder and $L^{p}$ estimates for parabolic systems via Campanato spaces. Commun. Partial Differ. Equ. 21(7-8), 1141-1175 (1996)

38. Spivak, M.: A Comprehensive Introduction to Differential Geometry, vol. IV. Publish or Perish, Inc., Boston (1975)

39. Spivak, M.: A Comprehensive Introduction to Differential Geometry, vol. II, 2nd edn. Publish or Perish Inc, Wilmington (1979)

40. Tang, B., Sapiro, G., Caselles, V.: Diffusion of general data on non-flat manifolds via harmonic maps theory: the direction diffusion case. Int. J. Comput. Vis. 36(2), 149-161 (2000)

41. Tang, B., Sapiro, G., Caselles, V.: Color image enhancement via chromaticity diffusion. IEEE Trans. Image Process. 10(5), 701-707 (2001)

42. Temam, R.: Problèmes mathématiques en plasticité. Méthodes Mathématiques de l'Informatique [Mathematical Methods of Information Science], vol. 12. Gauthier-Villars, Montrouge (1983)

43. Tu, L.W.: An Introduction to Manifolds: Universitext, 2nd edn. Springer, New York (2011)

44. Vespri, V.: Local existence, uniqueness and regularity for a class of degenerate parabolic systems arising in biological models. Rend. Sem. Mat. Univ. Padova 87, 209-244 (1992)

45. Weinmann, A., Demaret, L., Storath, M.: Total variation regularization for manifold-valued data. SIAM J. Imaging Sci. 7(4), 2226-2257 (2014)

Publisher's Note Springer Nature remains neutral with regard to jurisdictional claims in published maps and institutional affiliations. 